MATHEMATICS OF COMPUTATION

Volume 70, Number 236, Pages 1461-1480

S $0025-5718(00) 01267-9$

Article electronically published on May 23, 2000

\title{
A DUAL-DUAL MIXED FORMULATION FOR NONLINEAR EXTERIOR TRANSMISSION PROBLEMS
}

\author{
GABRIEL N. GATICA AND SALIM MEDDAHI
}

Dedicated to Professor Dr. George C. Hsiao on the occasion of his 65th birthday

\begin{abstract}
We combine a dual-mixed finite element method with a Dirichletto-Neumann mapping (derived by the boundary integral equation method) to study the solvability and Galerkin approximations of a class of exterior nonlinear transmission problems in the plane. As a model problem, we consider a nonlinear elliptic equation in divergence form coupled with the Laplace equation in an unbounded region of the plane. Our combined approach leads to what we call a dual-dual mixed variational formulation since the main operator involved has itself a dual-type structure. We establish existence and uniqueness of solution for the continuous and discrete formulations, and provide the corresponding error analysis by using Raviart-Thomas elements. The main tool of our analysis is given by a generalization of the usual Babuska-Brezzi theory to a class of nonlinear variational problems with constraints.
\end{abstract}

\section{INTRODUCTION}

The numerical solution of interior and exterior nonlinear-linear transmission problems usually combines the finite element method (FEM) in the nonlinear region with the boundary integral equation method (BIM) in the linear and homogeneous domain. This method, which is known as the coupling of FEM and BIM, has been applied successfully during the last decades using traditional finite elements and, more recently, using mixed finite elements as well (see, e.g., [3], [6], 16], 17, 18, 20], [21, 31], 34, and the references therein).

An alternative procedure for dealing with exterior problems consists of employing Dirichlet-to-Neumann mappings. This means that one first introduces a sufficiently large circle $\Gamma$ (in $\mathbf{R}^{2}$ ) or a sphere (in $\mathbf{R}^{3}$ ), so that the linear domain is divided into a bounded annular region and an unbounded one. Next, one derives an explicit formula for the Neumann data on $\Gamma$ in terms of the Dirichlet data on the same curve, which is known as the Dirichlet-to-Neumann mapping. This has been done for several elliptic operators, including the Lamé system for elasticity, by using Fouriertype series developments (see, e.g., [9], [23], [24], [25]). Then, in [11] we utilized the mapping obtained in 24] together with our mixed finite element approach from [21] to study the weak solvability of an exterior hyperelastic interface problem.

Received by the editor April 13, 1999 and, in revised form, October 13, 1999.

2000 Mathematics Subject Classification. Primary 65N30, 65N38, 65J15, 35J65, 35J05.

Key words and phrases. Mixed finite elements, boundary elements, coupling.

This research was partially supported by Fondecyt-Chile through research project 1980122, and by FONDAP-Conicyt through Program A on Numerical Analysis. 
Nevertheless, up to now, all the works on the combined use of mixed-FEM with either BIM or Dirichlet-to-Neumann mappings for nonlinear transmission problems have provided satisfactory results only at the continuous level. The associated Galerkin schemes still require some open questions to be solved. Indeed, in order to prove the unique solvability of the resulting variational formulations, one needs to introduce certain quotient spaces for which it is not clear how to define explicit finite element subspaces satisfying the corresponding discrete compatibility conditions. This drawback has motivated either the use of alternative mixed formulations (see, e.g., [1]) or the search of new tools from analysis to deal with the usual mixed formulations.

The purpose of the present paper is, precisely, to show some advances in the direction of the latter approach. In fact, we combine the dual-mixed finite element method from [20, 21] with a Dirichlet-to-Neumann mapping (derived by the BIM) to study the solvability and Galerkin approximation of a class of nonlinear exterior transmission problems in the plane. The resulting variational formulation can be written as what we call a dual-dual type operator equation, which, thanks to an extension of the usual Babuska-Brezzi theory, allows us to obtain satisfactory results for both the continuous and discrete schemes.

The rest of the paper is presented as follows. In Section 2, we describe the exterior transmission problem and transform it, using the Dirichlet-to-Neumann mapping, into a nonlocal boundary value problem on a bounded domain. The corresponding dual-dual mixed formulation is derived in Section 3. In Section 4, we recall the main results from a recent work concerning a generalization of the classical Babuska-Brezzi theory to a family of nonlinear variational problems with constraints. Finally, in Section 5 we apply the theorems from Section 4 and provide the existence and uniqueness of solution for the continuous and Galerkin dual-dual formulations by using Raviart-Thomas elements of lowest order. In addition, we prove the Cea estimate and provide, under usual regularity assumptions, an error bound of $O(h)$.

\section{THE EXTERIOR NONLINEAR TRANSMISSION PROBLEM}

Let $\Omega_{0}$ be a bounded and simply connected domain in $\mathbf{R}^{2}$ with Lipschitzcontinuous boundary $\Gamma_{0}$. Also, let $\Omega_{1}$ be the annular domain bounded by $\Gamma_{0}$ and another Lipschitz-continuous closed curve $\Gamma_{1}$ whose interior region contains $\Omega_{0}$. In addition, let $a_{i}: \Omega_{1} \times \mathbf{R}^{2} \rightarrow \mathbf{R}, i=1,2$, be nonlinear mappings satisfying certain conditions to be specified later on. Then, given $f_{1} \in L^{2}\left(\Omega_{1}\right)$, we consider the exterior nonlinear transmission problem: Find $u_{1} \in H^{1}\left(\Omega_{1}\right)$ and $u_{2} \in H_{\mathrm{loc}}^{1}\left(\mathbf{R}^{2}-\bar{\Omega}_{0} \cup \bar{\Omega}_{1}\right)$ such that

$$
\begin{gathered}
u_{1}=0 \quad \text { on } \Gamma_{0}, \\
-\sum_{i=1}^{2} \frac{\partial}{\partial x_{i}} a_{i}\left(\cdot, \nabla u_{1}(\cdot)\right)=f_{1} \quad \text { in } \Omega_{1}, \\
u_{1}=u_{2} \text { and } \sum_{i=1}^{2} a_{i}\left(\cdot, \nabla u_{1}(\cdot)\right) n_{i}-\frac{\partial u_{2}}{\partial \mathbf{n}}=0 \text { on } \Gamma_{1}, \\
-\Delta u_{2}=0 \text { in } \mathbf{R}^{2}-\bar{\Omega}_{0} \cup \bar{\Omega}_{1}, \\
u_{2}(x)=O(1) \text { as }\|x\| \rightarrow+\infty,
\end{gathered}
$$

where $\mathbf{n}:=\left(n_{1}, n_{2}\right)$ denotes the unit outward normal to $\partial \Omega_{1}$. This kind of problem appears in the computation of magnetic fields of electromagnetic devices (see, e.g., 
[26, [27]), in some subsonic flow and fluid mechanics problems (see, e.g., [7, 8]), and also in steady heat conduction. For instance, in the latter case, one has $a_{i}(x, \nabla u(x))=k(x, \nabla u(x)) \frac{\partial u}{\partial x_{i}}$, where $u$ is the temperature and $k$ is the heat conductivity. In all these problems, and in many others from physics and engineering sciences, the fluxes become variables of much interest and are required, therefore, to be approximated directly. This fact motivates the use of mixed finite element formulations.

According to the above comment, in what follows we apply a dual-mixed finite element method in $\Omega_{1}$ and a Dirichlet-to-Neumann mapping (arising from the boundary integral equation method) in the exterior region $\mathbf{R}^{2}-\bar{\Omega}_{0} \cup \bar{\Omega}_{1}$. To this end, we first introduce a sufficiently large circle $\Gamma$ with center at the origin and radius $r$ such that its interior region contains $\bar{\Omega}_{0} \cup \bar{\Omega}_{1}$. We denote by $\Omega_{2}$ the annular region bounded by $\Gamma_{1}$ and $\Gamma$ and put $\Omega:=\Omega_{1} \cup \Gamma_{1} \cup \Omega_{2}$. Next, we define

$$
u:=\left\{\begin{array}{lll}
u_{1} & \text { in } & \Omega_{1}, \\
u_{2} & \text { in } & \Omega_{2},
\end{array}\right.
$$

the flux variable

$$
\boldsymbol{\sigma}:=\left\{\begin{array}{l}
\left(a_{1}(\cdot, \nabla u), a_{2}(\cdot, \nabla u)\right)^{T} \text { in } \Omega_{1}, \\
\nabla u \text { in } \Omega_{2},
\end{array}\right.
$$

the global data

$$
f:=\left\{\begin{array}{l}
f_{1} \text { in } \Omega_{1}, \\
0 \text { in } \Omega_{2},
\end{array}\right.
$$

and introduce the auxiliary unknowns

$$
\boldsymbol{\theta}:=\nabla u \quad \text { in } \quad \Omega \quad \text { and } \quad \xi:=\left.u\right|_{\Gamma} .
$$

On the other hand, by applying the boundary integral equation method in the region exterior to the circle $\Gamma$, and according to the analysis from [28] (see also [19]), we obtain the Dirichlet-to-Neumann mapping

$$
\frac{\partial u}{\partial \nu}=-2 \mathbf{W}\left(\left.u\right|_{\Gamma}\right) \quad \text { on } \Gamma, \quad \text { or equivalently, } \boldsymbol{\sigma} \cdot \nu=-2 \mathbf{W} \xi \text { on } \Gamma,
$$

where $\nu$ is the unit outward normal to $\Gamma$ and $\mathbf{W}$ is the hypersingular boundary integral operator associated with the Laplacian. Denoting by $\nu(z)$ the unit outward normal to $z \in \Gamma$, we have

$$
(\mathbf{W} \lambda)(x):=-\frac{\partial}{\partial \nu(x)} \int_{\Gamma}\left\{\frac{\partial}{\partial \nu(y)} E(x, y)\right\} \lambda(y) d s_{y} \quad \forall x \in \Gamma, \forall \lambda \in H^{1 / 2}(\Gamma),
$$

where $E(x, y):=-\frac{1}{2 \pi} \log \|x-y\|$ is the two-dimensional fundamental solution of the Laplace operator. It is well known (see, e.g., [5]) that $\mathbf{W}: H^{1 / 2}(\Gamma) \rightarrow H^{-1 / 2}(\Gamma)$ is linear and bounded and that there exists $C_{0}>0$ such that

$$
\langle\lambda, \mathbf{W} \lambda\rangle \geq C_{0}\|\lambda\|_{H^{1 / 2}(\Gamma)}^{2} \quad \forall \lambda \in H_{0}^{1 / 2}(\Gamma),
$$

where, hereafter, $\langle\cdot, \cdot\rangle$ denotes the duality pairing of $H^{1 / 2}(\Gamma)$ and $H^{-1 / 2}(\Gamma)$ with respect to the $L^{2}(\Gamma)$-inner product, and

$$
H_{0}^{1 / 2}(\Gamma):=\left\{\lambda \in H^{1 / 2}(\Gamma): \quad\langle\lambda, 1\rangle=0\right\} .
$$


Also, since $\mathbf{W} 1 \equiv 0$ on $\Gamma$, we note that it suffices to look for the unknown $\xi$ in the space $H_{0}^{1 / 2}(\Gamma)$.

Now, the asymptotic behaviour of $u$ at $\infty$ implies that $\int_{\Gamma} \frac{\partial u}{\partial \nu} d s=0$, which means that $\boldsymbol{\sigma} \in H_{0}(\operatorname{div} ; \Omega)$, where

$$
H_{0}(\operatorname{div} ; \Omega):=\{\boldsymbol{\tau} \in H(\operatorname{div} ; \Omega):\langle 1, \boldsymbol{\tau} \cdot \nu\rangle=0\} .
$$

We recall here that $H(\operatorname{div} ; \Omega)$ is the space of functions $\tau \in\left[L^{2}(\Omega)\right]^{2}$ such that $\operatorname{div} \boldsymbol{\tau} \in L^{2}(\Omega)$. Provided with the inner product $\langle\boldsymbol{\tau}, \boldsymbol{\sigma}\rangle_{H(\mathrm{div} ; \Omega)}:=\langle\boldsymbol{\tau}, \boldsymbol{\sigma}\rangle_{\left[L^{2}(\Omega)\right]^{2}}+$ $\langle\operatorname{div} \boldsymbol{\tau}, \operatorname{div} \boldsymbol{\sigma}\rangle_{L^{2}(\Omega)}, H(\operatorname{div} ; \Omega)$ is a Hilbert space. Moreover, for all $\boldsymbol{\tau} \in H(\operatorname{div} ; \Omega)$, $\boldsymbol{\tau} \cdot \nu \in H^{-1 / 2}(\Gamma)$ and $\|\boldsymbol{\tau} \cdot \nu\|_{H^{-1 / 2}(\Gamma)} \leq\|\boldsymbol{\tau}\|_{H(\operatorname{div} ; \Omega)}$ (see [22] for the proof of these results).

By virtue of the above analysis, the exterior transmission problem (2.1) can be reformulated as the following nonlocal boundary value problem in $\bar{\Omega}$ : Find $(\boldsymbol{\theta}, \xi, \boldsymbol{\sigma}, u) \in\left[L^{2}(\Omega)\right]^{2} \times H_{0}^{1 / 2}(\Gamma) \times H_{0}(\operatorname{div} ; \Omega) \times L^{2}(\Omega)$ such that

$$
\begin{aligned}
& u=0 \quad \text { on } \Gamma_{0}, \quad \boldsymbol{\theta}=\nabla u \text { in } \Omega, \\
& \boldsymbol{\sigma}=\left\{\begin{array}{lll}
a(\cdot, \boldsymbol{\theta}) & \text { in } \Omega_{1} \\
\boldsymbol{\theta} & \text { in } & \Omega_{2}
\end{array} \quad \text { and } \operatorname{div} \boldsymbol{\sigma}=-f \text { in } \Omega,\right. \\
& \boldsymbol{\sigma} \cdot \nu=-2 W \xi \text { and } u=\xi \text { on } \Gamma,
\end{aligned}
$$

where we have adopted the notation $a(\cdot, \boldsymbol{\theta}):=\left(a_{1}(\cdot, \boldsymbol{\theta}), a_{2}(\cdot, \boldsymbol{\theta})\right)^{T}$, and the second and fourth equations of (2.5) must be taken in the distributional sense.

The previous procedure induces a dual-mixed finite element approach in $\Omega$, which, up to now, is very close to the one employed in [20, 21] and [11]. However, the main difference will arise later on when we derive the corresponding variational formulation. Indeed, instead of using the complicated quotient spaces introduced in [20], we will rewrite the formulation in such a way that only the spaces indicated in (2.5) will be required in our subsequent analysis.

\section{The DUAL-DUAL MiXed FORMUlation}

From now on, we assume that the nonlinear mappings $a_{i}$ satisfy the following conditions:

(A.1) Carathéodory condition. The function $a_{i}(\cdot, \hat{\boldsymbol{\theta}}), i=1,2$, is measurable in $\Omega_{1}$ for all $\hat{\boldsymbol{\theta}} \in \mathbf{R}^{2}$, and $a_{i}(x, \cdot)$ is continuous in $\mathbf{R}^{2}$ for almost all $x \in \Omega_{1}$.

(A.2) Growth condition. There exist functions $\phi_{i} \in L^{2}\left(\Omega_{1}\right), i=1,2$, such that

$$
\left|a_{i}(x, \hat{\boldsymbol{\theta}})\right| \leq C\{1+|\hat{\boldsymbol{\theta}}|\}+\left|\phi_{i}(x)\right|,
$$

for all $\hat{\boldsymbol{\theta}} \in \mathbf{R}^{2}$ and for almost all $x \in \Omega_{1}$.

(A.3) The functions $a_{i}(x, \cdot), i=1,2$, have continuous first order partial derivatives in $\mathbf{R}^{2}$ for almost all $x \in \Omega_{1}$. In addition, there exists $C>0$ such that

$$
\sum_{i, j=1}^{2} \frac{\partial}{\partial \hat{\theta}_{j}} a_{i}(x, \hat{\boldsymbol{\theta}}) \hat{\zeta}_{i} \hat{\zeta}_{j} \geq C \sum_{i=1}^{2} \hat{\zeta}_{i}^{2},
$$

for all $\hat{\boldsymbol{\theta}}:=\left(\hat{\theta}_{1}, \hat{\theta}_{2}\right), \hat{\boldsymbol{\zeta}}:=\left(\hat{\zeta}_{1}, \hat{\zeta}_{2}\right) \in \mathbf{R}^{2}$ and for almost all $x \in \Omega_{1}$. 
(A.4) The functions $a_{i}(x, \cdot)$ have continuous first order partial derivatives in $\mathbf{R}^{2}$ for almost all $x \in \Omega_{1}$. In addition, there exists $C>0$ such that for each $i, j \in\{1,2\}$, $\frac{\partial}{\partial \hat{\theta}_{j}} a_{i}(x, \hat{\boldsymbol{\theta}})$ satisfies the Carathéodory condition (A.1), and $\left|\frac{\partial}{\partial \hat{\theta}_{j}} a_{i}(x, \hat{\boldsymbol{\theta}})\right| \leq C$, for all $\hat{\boldsymbol{\theta}} \in \mathbf{R}^{2}$ and for almost all $x \in \Omega_{1}$.

For specific examples of coefficients $a_{i}$ satisfying the above conditions, we refer to [4, 34] and 35].

As a consequence of (A.1) and (A.2), one can prove (see, e.g., Theorem 2.8 in [10]) that the Nemytsky operator $\mathcal{A}_{i}:\left[L^{2}\left(\Omega_{1}\right)\right]^{2} \rightarrow L^{2}\left(\Omega_{1}\right)$, defined by $\left(\mathcal{A}_{i} \boldsymbol{\theta}\right)(x):=$ $a_{i}(x, \boldsymbol{\theta}(x))$ for all $\boldsymbol{\theta} \in\left[L^{2}\left(\Omega_{1}\right)\right]^{2}$ and for almost all $x \in \Omega_{1}$, is continuous and bounded.

Now, for the weak formulation, we first multiply the second equation in (2.5) by a function $\tau \in H_{0}(\operatorname{div} ; \Omega)$, integrate by parts in $\Omega$, and use that $u=0$ on $\Gamma_{0}$ and that $u=\xi$ on $\Gamma$, to obtain

$$
-\int_{\Omega} \boldsymbol{\theta} \cdot \boldsymbol{\tau} d x+\langle\xi, \boldsymbol{\tau} \cdot \nu\rangle-\int_{\Omega} u \operatorname{div} \boldsymbol{\tau} d x=0 .
$$

Next, the third equation in (2.5) is tested against $\zeta \in\left[L^{2}(\Omega)\right]^{2}$, which gives

$$
\int_{\Omega_{1}} a(\cdot, \boldsymbol{\theta}) \cdot \boldsymbol{\zeta} d x+\int_{\Omega_{2}} \boldsymbol{\theta} \cdot \boldsymbol{\zeta} d x-\int_{\Omega} \boldsymbol{\sigma} \cdot \boldsymbol{\zeta} d x=0 .
$$

Finally, the fourth and fifth equations in (2.5) are tested against $v \in L^{2}(\Omega)$ and $\lambda \in H_{0}^{1 / 2}(\Gamma)$, respectively, which yields

$$
-\int_{\Omega} v \operatorname{div} \boldsymbol{\sigma} d x=\int_{\Omega} f v d x
$$

and

$$
2\langle\lambda, W \xi\rangle+\langle\lambda, \boldsymbol{\sigma} \cdot \nu\rangle=0
$$

Thus, collecting appropriately (3.1), (3.2), (3.3) and (3.4), we arrive at the following variational formulation of (2.5) $)$ : Find $((\boldsymbol{\theta}, \xi), \boldsymbol{\sigma}, u) \in\left(\left[L^{2}(\Omega)\right]^{2} \times H_{0}^{1 / 2}(\Gamma)\right) \times$ $H_{0}(\operatorname{div} ; \Omega) \times L^{2}(\Omega)$ such that

$$
\begin{aligned}
\int_{\Omega_{1}} a(\cdot, \boldsymbol{\theta}) \cdot \boldsymbol{\zeta} d x+\int_{\Omega_{2}} \boldsymbol{\theta} \cdot \boldsymbol{\zeta} d x+2\langle\lambda, \mathbf{W} \xi\rangle & \\
-\int_{\Omega} \boldsymbol{\sigma} \cdot \boldsymbol{\zeta} d x+\langle\lambda, \boldsymbol{\sigma} \cdot \nu\rangle & =0 \\
-\int_{\Omega} \boldsymbol{\theta} \cdot \boldsymbol{\tau} d x+\langle\xi, \boldsymbol{\tau} \cdot \nu\rangle-\int_{\Omega} u \operatorname{div} \boldsymbol{\tau} d x & =0 \\
-\int_{\Omega} v \operatorname{div} \boldsymbol{\sigma} d x & =\int_{\Omega} f v d x,
\end{aligned}
$$

for all $((\boldsymbol{\zeta}, \lambda), \boldsymbol{\tau}, v) \in\left(\left[L^{2}(\Omega)\right]^{2} \times H_{0}^{1 / 2}(\Gamma)\right) \times H_{0}(\operatorname{div} ; \Omega) \times L^{2}(\Omega)$.

We show next that 3.5 can be rewritten in the form of a nonlinear variational problem with linear constraints. For this purpose, we put $X_{1}:=\left[L^{2}(\Omega)\right]^{2} \times H_{0}^{1 / 2}(\Gamma)$, $M_{1}:=H_{0}(\operatorname{div} ; \Omega), X:=X_{1} \times M_{1}, M:=L^{2}(\Omega)$, denote $\mathbf{t}:=(\boldsymbol{\theta}, \xi), \mathbf{s}:=(\boldsymbol{\zeta}, \lambda) \in X_{1}$, 
and define the operators $\mathbf{A}_{1}: X_{1} \rightarrow X_{1}^{\prime}, \mathbf{B}_{1}: X_{1} \rightarrow M_{1}^{\prime}, \mathbf{A}: X \rightarrow X^{\prime}, \mathbf{B}: X \rightarrow M^{\prime}$, and the functional $\mathbf{G} \in M^{\prime}$, as follows:

$$
\begin{gathered}
{\left[\mathbf{A}_{1}(\mathbf{t}), \mathbf{s}\right]:=\int_{\Omega_{1}} a(\cdot, \boldsymbol{\theta}) \cdot \boldsymbol{\zeta} d x+\int_{\Omega_{2}} \boldsymbol{\theta} \cdot \boldsymbol{\zeta} d x+2\langle\lambda, \mathbf{W} \xi\rangle,} \\
{\left[\mathbf{B}_{1}(\mathbf{t}), \boldsymbol{\tau}\right]:=-\int_{\Omega} \boldsymbol{\theta} \cdot \boldsymbol{\tau} d x+\langle\xi, \boldsymbol{\tau} \cdot \nu\rangle,} \\
{[\mathbf{A}(\mathbf{t}, \boldsymbol{\sigma}),(\mathbf{s}, \boldsymbol{\tau})]:=\left[\mathbf{A}_{1}(\mathbf{t}), \mathbf{s}\right]+\left[\mathbf{B}_{1}(\mathbf{s}), \boldsymbol{\sigma}\right]+\left[\mathbf{B}_{1}(\mathbf{t}), \boldsymbol{\tau}\right],} \\
{[\mathbf{B}(\mathbf{t}, \boldsymbol{\sigma}), v]:=-\int_{\Omega} v \operatorname{div} \boldsymbol{\sigma} d x}
\end{gathered}
$$

and

$$
[\mathbf{G}, v]:=\int_{\Omega} f v d x
$$

for all $(\mathbf{t}, \boldsymbol{\sigma}),(\mathbf{s}, \boldsymbol{\tau}) \in X$ and for all $v \in M$, where $[\cdot, \cdot]$ stands for the duality pairing induced by the operators appearing in each case.

Further, let $\mathbf{B}_{1}^{*}: M_{1} \rightarrow X_{1}^{\prime}$ and $\mathbf{B}^{*}: M \rightarrow X^{\prime}$ be the transposes of $\mathbf{B}_{1}$ and $\mathbf{B}$, respectively, and let $\mathbf{O}$ denote both the null functional and the null operator.

It is worth remarking that $\mathbf{B}_{1}$ and $\mathbf{B}$ are linear and bounded operators, and that $\mathbf{A}_{1}$, and hence $\mathbf{A}$, are nonlinear. Moreover, $\mathbf{A}$ can be defined, equivalently, as:

$$
\mathbf{A}(\mathbf{t}, \boldsymbol{\sigma}):=\left[\begin{array}{cc}
\mathbf{A}_{1} & \mathbf{B}_{1}^{*} \\
\mathbf{B}_{1} & \mathbf{O}
\end{array}\right]\left[\begin{array}{c}
\mathbf{t} \\
\boldsymbol{\sigma}
\end{array}\right] \in X^{\prime}:=X_{1}^{\prime} \times M_{1}^{\prime} .
$$

Therefore, the system (3.5) can be reformulated as the following operator equation: Find $((\mathbf{t}, \boldsymbol{\sigma}), u) \in X \times M$ such that

$$
\left[\begin{array}{ll}
\mathbf{A} & \mathbf{B}^{*} \\
\mathbf{B} & \mathbf{O}
\end{array}\right]\left[\begin{array}{c}
(\mathbf{t}, \boldsymbol{\sigma}) \\
u
\end{array}\right]=\left[\begin{array}{l}
\mathbf{O} \\
\mathbf{G}
\end{array}\right] .
$$

The equation (3.12), which can be viewed as a nonlinear variational problem with linear constraints, constitutes our so-called dual-dual mixed formulation of (2.5) since the operator $\mathbf{A}$ itself has the dual-type structure given by (3.11).

In order to establish the unique solvability of (3.12), study its Galerkin approximations, and derive the corresponding error analysis, we need an extension of the usual Babuska-Brezzi theory to the above class of nonlinear problems. This is, precisely, the subject of the next section. We will go back to our problem (3.12) in Section 5 .

\section{An extension of the Babuska-Brezzi theory}

In the recent paper [12] we have generalized the classical Babuska-Brezzi theory to the class of nonlinear variational problems with constraints given by (3.12). The purpose of this section is to recall the main results from that work.

In order to set the abstract problem of interest, we let $X_{1}, M_{1}, M$ be Hilbert spaces and define $X:=X_{1} \times M_{1}$. Then, we consider a nonlinear operator $\mathbf{A}_{1}$ : $X_{1} \rightarrow X_{1}^{\prime}$, and linear bounded operators $\mathbf{B}_{1}: X_{1} \rightarrow M_{1}^{\prime}$ and $\mathbf{B}: X \rightarrow M^{\prime}$, with transposes $\mathbf{B}_{1}^{*}: M_{1} \rightarrow X_{1}^{\prime}$ and $\mathbf{B}^{*}: M \rightarrow X^{\prime}$, respectively. With $\mathbf{A}_{1}, \mathbf{B}_{1}$ and $\mathbf{B}_{1}^{*}$ we define a nonlinear operator $\mathbf{A}: X \rightarrow X^{\prime}$ as in (3.11). 
Then, we are interested in the following nonlinear variational problem: Given $(\mathbf{F}, \mathbf{G}) \in X^{\prime} \times M^{\prime}$, find $((\mathbf{t}, \boldsymbol{\sigma}), u) \in X \times M$ such that

$$
\left[\begin{array}{ll}
\mathbf{A} & \mathbf{B}^{*} \\
\mathbf{B} & \mathbf{O}
\end{array}\right]\left[\begin{array}{c}
(\mathbf{t}, \boldsymbol{\sigma}) \\
u
\end{array}\right]=\left[\begin{array}{l}
\mathbf{F} \\
\mathbf{G}
\end{array}\right] \text {. }
$$

Sufficient conditions for the unique solvability of (4.1) are provided in the following theorem.

Theorem 4.1. Let $V:=\operatorname{Ker}(\mathbf{B})$ such that $V=\tilde{X}_{1} \times \tilde{M}_{1}$, with $\tilde{X}_{1} \subseteq X_{1}$ and $\tilde{M}_{1} \subseteq M_{1}$. Also, define $V_{1}:=\left\{\mathbf{s} \in \tilde{X}_{1}:\left[\mathbf{B}_{1}(\mathbf{s}), \boldsymbol{\tau}\right]=0 \forall \boldsymbol{\tau} \in \tilde{M}_{1}\right\}$ and let $\Pi_{1}$ : $X_{1}^{\prime} \rightarrow V_{1}^{\prime}$ be the canonical imbedding defined by $\Pi_{1}\left(\mathbf{F}_{1}\right)=\left.\mathbf{F}_{1}\right|_{V_{1}}$ for all $\mathbf{F}_{1} \in X_{1}^{\prime}$. Assume that

i) there exists $\beta>0$ such that for all $v \in M$

$$
\sup _{\substack{(\mathbf{s}, \boldsymbol{\tau}) \in X \\(\mathbf{s}, \boldsymbol{\tau}) \neq 0}} \frac{[\mathbf{B}(\mathbf{s}, \boldsymbol{\tau}), v]}{\|(\mathbf{s}, \boldsymbol{\tau})\|_{X}} \geq \beta\|v\|_{M} ;
$$

ii) there exists $\beta_{1}>0$ such that for all $\boldsymbol{\tau} \in \tilde{M}_{1}$

$$
\sup _{\substack{\mathbf{s} \in \tilde{X}_{1} \\ \mathbf{s} \neq 0}} \frac{\left[\mathbf{B}_{1}(\mathbf{s}), \boldsymbol{\tau}\right]}{\|\mathbf{s}\|_{X_{1}}} \geq \beta_{1}\|\boldsymbol{\tau}\|_{M_{1}}
$$

iii) the nonlinear operator $\mathbf{A}_{1}: X_{1} \rightarrow X_{1}^{\prime}$ is Lipschitz continuous with a Lipschitz constant $\gamma>0$, and for any $\tilde{\mathbf{t}} \in X_{1}$, the nonlinear operator $\Pi_{1} \mathbf{A}_{1}(\cdot+\tilde{\mathbf{t}})$ : $V_{1} \rightarrow V_{1}^{\prime}$ is strongly monotone.

Then, for each $(\mathbf{F}, \mathbf{G}) \in X^{\prime} \times M^{\prime}$ there exists a unique $((\mathbf{t}, \boldsymbol{\sigma}), u) \in X \times M$ solution of (4.1).

Proof. We adapt the analysis from [22] (Chapter I, Section 4) to the present situation. Thus, given $\mathbf{G} \in M^{\prime}$ we set

$$
V(\mathbf{G}):=\{(\mathbf{s}, \boldsymbol{\tau}) \in X: \quad \mathbf{B}(\mathbf{s}, \boldsymbol{\tau})=\mathbf{G}\}
$$

and observe that $V:=\operatorname{Ker}(\mathbf{B})=V(\mathbf{O})$.

Then, with (4.1) we associate the following problem: Find $(\mathbf{t}, \boldsymbol{\sigma}) \in V(\mathbf{G})$ such that

$$
[\mathbf{A}(\mathbf{t}, \boldsymbol{\sigma}),(\mathbf{s}, \boldsymbol{\tau})]=[\mathbf{F},(\mathbf{s}, \boldsymbol{\tau})] \quad \forall(\mathbf{s}, \boldsymbol{\tau}) \in V .
$$

Clearly, if $(\mathbf{t}, \boldsymbol{\sigma}) \in V(\mathbf{G})$ is a solution of 4.2$)$, then, due to the inf-sup condition Theorem $4.1 \mathrm{i}$ ) and Lemma 4.1 in Chapter I of [22], there exists a unique $u \in M$ such that $((\mathbf{t}, \boldsymbol{\sigma}), u) \in X \times M$ is a solution of (4.1).

Conversely, if $((\mathbf{t}, \boldsymbol{\sigma}), u) \in X \times M$ is a solution of (4.1), then $(\mathbf{t}, \boldsymbol{\sigma}) \in V(\mathbf{G})$ and $(\mathbf{t}, \boldsymbol{\sigma})$ is a solution of (4.2) since for all $(\mathbf{s}, \boldsymbol{\tau}) \in V,\left[\mathbf{B}^{*}(u),(\mathbf{s}, \boldsymbol{\tau})\right]=[\mathbf{B}(\mathbf{s}, \boldsymbol{\tau}), u]=0$.

Because of this equivalence, we now concentrate on problem (4.2). Again, by Lemma 4.1 in Chapter I of [22, there exists $\left(\mathbf{t}_{0}, \boldsymbol{\sigma}_{0}\right) \in X$ such that $\mathbf{B}\left(\mathbf{t}_{0}, \boldsymbol{\sigma}_{0}\right)=\mathbf{G}$. Thus, problem (4.2) can be replaced by: Find $(\tilde{\mathbf{t}}, \tilde{\boldsymbol{\sigma}}) \in V$ such that

$$
\left[\mathbf{A}_{1}\left(\tilde{\mathbf{t}}+\mathbf{t}_{0}\right), \mathbf{s}\right]+\left[\mathbf{B}_{1}^{*}(\tilde{\boldsymbol{\sigma}}), \mathbf{s}\right]=\left[\tilde{\mathbf{F}}_{1}, \mathbf{s}\right]
$$

$$
\left[\mathbf{B}_{1}(\tilde{\mathbf{t}}), \boldsymbol{\tau}\right]=\left[\tilde{\mathbf{G}}_{1}, \boldsymbol{\tau}\right]
$$


for all $(\mathbf{s}, \boldsymbol{\tau}) \in V$, where $\tilde{\mathbf{F}}_{1}:=\mathbf{F}_{1}-\mathbf{B}_{1}^{*}\left(\boldsymbol{\sigma}_{0}\right) \in X_{1}^{\prime}$ and $\tilde{\mathbf{G}}_{1}:=\mathbf{G}_{1}-\mathbf{B}_{1}\left(\mathbf{t}_{0}\right) \in M_{1}^{\prime}$, with $\mathbf{F}:=\left(\mathbf{F}_{1}, \mathbf{G}_{1}\right) \in X_{1}^{\prime} \times M_{1}^{\prime}=: X^{\prime}$.

Next, we set

$$
V_{1}\left(\tilde{\mathbf{G}}_{1}\right):=\left\{\mathbf{s} \in \tilde{X}_{1}: \quad\left[\mathbf{B}_{1}(\mathbf{s}), \boldsymbol{\tau}\right]=\left[\tilde{\mathbf{G}}_{1}, \boldsymbol{\tau}\right] \quad \forall \boldsymbol{\tau} \in \tilde{M}_{1}\right\}
$$

and observe that $V_{1}=V_{1}(\mathbf{O})$.

Then we associate with (4.3) the following problem: Find $\tilde{\mathbf{t}} \in V_{1}\left(\tilde{\mathbf{G}}_{1}\right)$ such that

$$
\left[\mathbf{A}_{1}\left(\tilde{\mathbf{t}}+\mathbf{t}_{0}\right), \mathbf{s}\right]=\left[\tilde{\mathbf{F}}_{1}, \mathbf{s}\right] \quad \forall \mathbf{s} \in V_{1} .
$$

Using Theorem $4.1 \mathrm{ii}$ ) and Lemma 4.1 in Chapter I of [22], we deduce that there exists $\tilde{\mathbf{t}}_{0} \in \tilde{X}_{1}$ such that $\left[\mathbf{B}_{1}\left(\tilde{\mathbf{t}}_{0}\right), \boldsymbol{\tau}\right]=\left[\tilde{\mathbf{G}}_{1}, \boldsymbol{\tau}\right]$ for all $\boldsymbol{\tau} \in \tilde{M}_{1}$. Therefore, problem (4.4) can be replaced by: Find $\hat{\mathbf{t}} \in V_{1}$ such that

$$
\left[\mathbf{A}_{1}\left(\hat{\mathbf{t}}+\tilde{\mathbf{t}}_{0}+\mathbf{t}_{0}\right), \mathbf{s}\right]=\left[\tilde{\mathbf{F}}_{1}, \mathbf{s}\right] \quad \forall \mathbf{s} \in V_{1} .
$$

Thus, due to the hypotheses on $\mathbf{A}_{1}$ (see Theorem 4.1 iii)) and thanks to a well known result from nonlinear functional analysis (see, e.g., Theorem 3.3.23 in [32]) we conclude that (4.5) has a unique solution $\hat{\mathbf{t}} \in V_{1}$, and hence $\tilde{\mathbf{t}}:=\hat{\mathbf{t}}+\tilde{\mathbf{t}}_{0} \in V_{1}\left(\tilde{\mathbf{G}}_{1}\right)$ is the unique solution of (4.4). It follows, in virtue of Theorem $4.1 \mathrm{ii})$ and Lemma 4.1 in Chapter I of [22, that there exists $\tilde{\boldsymbol{\sigma}} \in M_{1}$ such that $(\tilde{\mathbf{t}}, \tilde{\boldsymbol{\sigma}}) \in V$ is the unique solution of (4.3). In this way, we deduce that $(\mathbf{t}, \boldsymbol{\sigma}):=\left(\tilde{\mathbf{t}}+\mathbf{t}_{0}, \tilde{\boldsymbol{\sigma}}+\boldsymbol{\sigma}_{0}\right) \in V(\mathbf{G})$ is the unique solution of (4.2). Finally, the equivalence between (4.1) and (4.2) completes the proof.

Now, for the Galerkin approximation of (4.1), we let $X_{1, h}, M_{1, h}$ and $M_{h}$ be finite dimensional subspaces of $X_{1}, M_{1}$ and $M$, respectively, and let $X_{h}:=X_{1, h} \times M_{1, h}$ be the corresponding subspace of $X$. Here, we assume that the index $h$ is taken in a numerable family $\mathcal{I}:=\left\{h_{j}\right\}_{j \in \mathbf{N}}$ such that $h_{j} \geq h_{j+1}$ for all $j \in \mathbf{N}$.

Thus, the Galerkin scheme associated with (4.1) reads as follows: $\operatorname{Given}(\mathbf{F}, \mathbf{G}) \in$ $X^{\prime} \times M^{\prime}$, find $\left(\left(\mathbf{t}_{h}, \boldsymbol{\sigma}_{h}\right), u_{h}\right) \in X_{h} \times M_{h}$ such that

$$
\begin{aligned}
{\left[\mathbf{A}\left(\mathbf{t}_{h}, \boldsymbol{\sigma}_{h}\right),\left(\mathbf{s}_{h}, \boldsymbol{\tau}_{h}\right)\right]+\left[\mathbf{B}^{*}\left(u_{h}\right),\left(\mathbf{s}_{h}, \boldsymbol{\tau}_{h}\right)\right] } & =\left[\mathbf{F},\left(\mathbf{s}_{h}, \boldsymbol{\tau}_{h}\right)\right], \\
{\left[\mathbf{B}\left(\mathbf{t}_{h}, \boldsymbol{\sigma}_{h}\right), v_{h}\right] } & =\left[\mathbf{G}, v_{h}\right],
\end{aligned}
$$

for all $\left(\left(\mathbf{s}_{h}, \boldsymbol{\tau}_{h}\right), v_{h}\right) \in X_{h} \times M_{h}$.

The discrete analogue of Theorem 4.1 is stated next.

Theorem 4.2. Let $V_{h}:=\left\{\left(\mathbf{s}_{h}, \boldsymbol{\tau}_{h}\right) \in X_{h}:\left[\mathbf{B}\left(\mathbf{s}_{h}, \boldsymbol{\tau}_{h}\right), v_{h}\right]=0 \forall v_{h} \in M_{h}\right\}$ such that $V_{h}:=\tilde{X}_{1, h} \times \tilde{M}_{1, h}$, with $\tilde{X}_{1, h} \subseteq X_{1, h}$ and $\tilde{M}_{1, h} \subseteq M_{1, h}$. Also, define $V_{1, h}:=$ $\left\{\mathbf{s}_{h} \in \tilde{X}_{1, h}:\left[\mathbf{B}_{1}\left(\mathbf{s}_{h}\right), \boldsymbol{\tau}_{h}\right]=0 \forall \boldsymbol{\tau}_{h} \in \tilde{M}_{1, h}\right\}$ and let $\Pi_{1, h}: X_{1, h}^{\prime} \rightarrow V_{1, h}^{\prime}$ be the canonical imbedding. Further, let $\mathbf{A}_{1, h}:=p_{h}^{\prime} \mathbf{A}_{1}: X_{1} \rightarrow X_{1, h}^{\prime}$ where $p_{h}: X_{1, h} \rightarrow X_{1}$ is the canonical injection with adjoint $p_{h}^{\prime}: X_{1}^{\prime} \rightarrow X_{1, h}^{\prime}$. Assume that

i) there exists $\beta^{*}>0$, independent of the subspaces involved, such that for all $v_{h} \in M_{h}$

$$
\sup _{\substack{\left(\mathbf{s}_{h}, \boldsymbol{\tau}_{h}\right) \in X_{h} \\\left(\mathbf{s}_{h}, \boldsymbol{\tau}_{h}\right) \neq 0}} \frac{\left[\mathbf{B}\left(\mathbf{s}_{h}, \boldsymbol{\tau}_{h}\right), v_{h}\right]}{\left\|\left(\mathbf{s}_{h}, \boldsymbol{\tau}_{h}\right)\right\|_{X}} \geq \beta^{*}\left\|v_{h}\right\|_{M}
$$


ii) there exists $\beta_{1}^{*}>0$, independent of the subspaces involved, such that for all $\tau_{h} \in \tilde{M}_{1, h}$

$$
\sup _{\substack{\mathbf{s}_{h} \in \tilde{X}_{1, h} \\ \mathbf{s}_{h} \neq 0}} \frac{\left[\mathbf{B}_{1}\left(\mathbf{s}_{h}\right), \boldsymbol{\tau}_{h}\right]}{\left\|\mathbf{s}_{h}\right\|_{X_{1}}} \geq \beta_{1}^{*}\left\|\boldsymbol{\tau}_{h}\right\|_{M_{1}}
$$

iii) the nonlinear operator $\mathbf{A}_{1, h}: X_{1} \rightarrow X_{1, h}^{\prime}$ is Lipschitz-continuous, and for any $\tilde{\mathbf{t}} \in X_{1, h}$, the nonlinear operator $\Pi_{1, h} \mathbf{A}_{1, h}(\cdot+\tilde{\mathbf{t}}): V_{1, h} \rightarrow V_{1, h}^{\prime}$ is strongly monotone with a monotonicity constant $\alpha_{h}>0$ independent of $\tilde{\mathbf{t}}$.

Then, for each $(\mathbf{F}, \mathbf{G}) \in X^{\prime} \times M^{\prime}$ there exists a unique $\left(\left(\mathbf{t}_{h}, \boldsymbol{\sigma}_{h}\right), u_{h}\right) \in X_{h} \times M_{h}$ solution of (4.6).

Proof. It is similar to the proof of Theorem 4.1 and hence we omit further details. We refer the interested reader to Section 3 and Theorem 3.2 in [12].

Clearly, the Lipschitz-continuity of $\mathbf{A}_{1}$ yields the same property for $\mathbf{A}_{1, h}$, with the same Lipschitz constant $\gamma$, independent of $h$, given in Theorem 4.1.

Finally, concerning the error analysis, we recall the following result from [12].

Theorem 4.3. Assume that all the hypotheses of both Theorem 4.1 and Theorem 4.2 are satisfied, and let $((\mathbf{t}, \boldsymbol{\sigma}), u) \in X \times M$ and $\left(\left(\mathbf{t}_{h}, \boldsymbol{\sigma}_{h}\right), u_{h}\right) \in X_{h} \times M_{h}$ be the unique solutions of (4.1) and (4.6), respectively. Let $\mathbf{F}:=\left(\mathbf{F}_{1}, \mathbf{G}_{1}\right) \in X^{\prime}$, with $\mathbf{F}_{1} \in X_{1}^{\prime}$ and $\mathbf{G}_{1} \in M_{1}^{\prime}$. In addition, suppose that the family of nonlinear operators $\left\{\Pi_{1, h} \mathbf{A}_{1, h}(\cdot+\tilde{\mathbf{t}}): \quad \tilde{\mathbf{t}} \in X_{1, h}, h \in \mathcal{I}\right\}$ is uniformly strongly monotone, i.e., there exists $\alpha>0$ such that $\alpha_{h} \geq \alpha$ for all $h \in \mathcal{I}$. Then, there exists $C>0$, depending only on $\alpha, \gamma,\left\|\mathbf{B}_{1}\right\|, \beta_{1}^{*},\|\mathbf{B}\|$ and $\beta^{*}$, such that the following Strang-type error estimate holds for all $h \in \mathcal{I}$ :

$$
\begin{aligned}
&\left\|((\mathbf{t}, \boldsymbol{\sigma}), u)-\left(\left(\mathbf{t}_{h}, \boldsymbol{\sigma}_{h}\right), u_{h}\right)\right\| \\
& \leq C\{ \inf _{\left(\left(\mathbf{s}_{h}, \boldsymbol{\tau}_{h}\right), v_{h}\right) \in X_{h} \times M_{h}}\left\|((\mathbf{t}, \boldsymbol{\sigma}), u)-\left(\left(\mathbf{s}_{h}, \boldsymbol{\tau}_{h}\right), v_{h}\right)\right\| \\
&+\sup _{\substack{\tilde{s}_{h} \in \tilde{x}_{1, h} \\
\tilde{\mathbf{s}}_{h} \neq 0}}\left\{\frac{\left[\mathbf{F}_{1}-\mathbf{A}_{1}(\mathbf{t})-\mathbf{B}_{1}^{*}(\boldsymbol{\sigma}), \tilde{\mathbf{s}}_{h}\right]}{\left\|\tilde{\mathbf{s}}_{h}\right\|}\right\} \\
&\left.+\sup _{\substack{\tilde{\boldsymbol{\tau}}_{h} \in \tilde{M}_{1, h} \\
\tilde{\boldsymbol{\tau}}_{h} \neq 0}}\left\{\frac{\left[\mathbf{G}_{1}-\mathbf{B}_{1}(\mathbf{t}), \tilde{\boldsymbol{\tau}}_{h}\right]}{\left\|\tilde{\boldsymbol{\tau}}_{h}\right\|}\right\}\right\} .
\end{aligned}
$$

Proof. We do not give full details here, but just sketch the main ideas. For the whole proof, we refer to Section 4 in [12].

First, the discrete inf-sup condition satisfied by $\mathbf{B}($ cf. Theorem4.2 $\mathrm{i})$ ) guarantees the existence of $\left(\mathbf{t}_{0, h}, \boldsymbol{\sigma}_{0, h}\right) \in X_{1, h} \times M_{1, h}$ such that $\left[\mathbf{B}\left(\mathbf{t}_{0, h}, \boldsymbol{\sigma}_{0, h}\right), v_{h}\right]=\left[\mathbf{G}, v_{h}\right]$ for all $v_{h} \in M_{h}$. 
Then, by using the properties of the operators $\mathbf{A}_{1}$ and $\mathbf{B}_{1}$, one proves that for all $h \in \mathcal{I}$

$$
\begin{aligned}
\left\|\mathbf{t}-\mathbf{t}_{h}\right\| \leq \frac{1}{\alpha} \sup _{\substack{\tilde{\mathbf{s}}_{h} \in \tilde{X}_{1, h} \\
\tilde{\mathbf{s}}_{h} \neq 0}}\left\{\frac{\left[\mathbf{F}_{1}-\mathbf{A}_{1}(\mathbf{t})-\mathbf{B}_{1}^{*}(\boldsymbol{\sigma}), \tilde{\mathbf{s}}_{h}\right]}{\left\|\tilde{\mathbf{s}}_{h}\right\|}\right\} \\
+\left(1+\frac{\gamma}{\alpha}\right) \inf _{\mathbf{s}_{h} \in V_{1, h}\left(\mathbf{G}_{1}\right)}\left\|\mathbf{t}-\mathbf{s}_{h}\right\| \\
+\frac{\left\|\mathbf{B}_{1}\right\|}{\alpha} \inf _{\tilde{\boldsymbol{\tau}}_{h} \in \tilde{M}_{1, h}}\left\|\boldsymbol{\sigma}-\left(\tilde{\boldsymbol{\tau}}_{h}+\boldsymbol{\sigma}_{0, h}\right)\right\|,
\end{aligned}
$$

where

$$
V_{1, h}\left(\mathbf{G}_{1}\right):=\left\{\mathbf{s}_{h} \in X_{1, h}: \quad\left[\mathbf{B}_{1}\left(\mathbf{s}_{h}\right), \boldsymbol{\tau}_{h}\right]=\left[\mathbf{G}_{1}, \boldsymbol{\tau}_{h}\right] \forall \boldsymbol{\tau}_{h} \in \tilde{M}_{1, h}\right\}
$$

Now, the discrete inf-sup condition for $\mathbf{B}_{1}$ (cf. Theorem 4.2 ii)) allows us to improve the bound provided by the second term on the right hand side of inequality (4.8). Indeed, we show that the following estimate holds for all $h \in \mathcal{I}$ :

$$
\begin{aligned}
& \inf _{\mathbf{s}_{h} \in V_{1, h}\left(\mathbf{G}_{1}\right)}\left\|\mathbf{t}-\mathbf{s}_{h}\right\| \leq\left(1+\frac{\left\|\mathbf{B}_{1}\right\|}{\beta_{1}^{*}}\right) \inf _{\tilde{\mathbf{s}}_{h} \in \tilde{X}_{1, h}}\left\|\mathbf{t}-\left(\tilde{\mathbf{s}}_{h}+\mathbf{t}_{0, h}\right)\right\| \\
& +\frac{1}{\beta_{1}^{*}} \sup _{\substack{\tilde{\boldsymbol{\tau}}_{h} \in \tilde{M}_{1, h} \\
\tilde{\boldsymbol{\tau}}_{h} \neq 0}}\left\{\frac{\left[\mathbf{G}_{1}-\mathbf{B}_{1}(\mathbf{t}), \tilde{\boldsymbol{\tau}}_{h}\right]}{\left\|\tilde{\boldsymbol{\tau}}_{h}\right\|}\right\} .
\end{aligned}
$$

Next, by applying again the properties of the operators $\mathbf{A}_{1}$ and $\mathbf{B}_{1}$, and also the discrete inf-sup condition for $\mathbf{B}_{1}$, we obtain the following upper bound for the error $\left\|\sigma-\sigma_{h}\right\|:$

$$
\begin{aligned}
\left\|\boldsymbol{\sigma}-\boldsymbol{\sigma}_{h}\right\| \leq \frac{1}{\beta_{1}^{*}} \sup _{\substack{\tilde{\mathbf{s}}_{h} \in \tilde{X}_{1, h} \\
\tilde{\mathbf{s}}_{h} \neq 0}}\left\{\frac{\left[\mathbf{F}_{1}-\mathbf{A}_{1}(\mathbf{t})-\mathbf{B}_{1}^{*}(\boldsymbol{\sigma}), \tilde{\mathbf{s}}_{h}\right]}{\left\|\tilde{\mathbf{s}}_{h}\right\|}\right\}+\frac{\gamma}{\beta_{1}^{*}}\left\|\mathbf{t}-\mathbf{t}_{h}\right\| \\
+\left(1+\frac{\left\|\mathbf{B}_{1}\right\|}{\beta_{1}^{*}}\right) \inf _{\substack{\tilde{\boldsymbol{\tau}}_{h} \in \tilde{M}_{1, h}\\
}}\left\|\boldsymbol{\sigma}-\left(\tilde{\boldsymbol{\tau}}_{h}+\boldsymbol{\sigma}_{0, h}\right)\right\| .
\end{aligned}
$$

Hence, as a consequence of (4.8), (4.9) and (4.10), and using also the discrete inf-sup condition for $\mathbf{B}$, we deduce that there exists $\tilde{C}>0$, depending only on $\alpha$, $\gamma,\left\|\mathbf{B}_{1}\right\|, \beta_{1}^{*},\|\mathbf{B}\|$ and $\beta^{*}$, such that for all $h \in \mathcal{I}$ :

$$
\begin{aligned}
\left\|(\mathbf{t}, \boldsymbol{\sigma})-\left(\mathbf{t}_{h}, \boldsymbol{\sigma}_{h}\right)\right\| \leq \tilde{C}\left\{\inf _{\left(\mathbf{s}_{h}, \boldsymbol{\tau}_{h}\right) \in X_{h}}\left\|(\mathbf{t}, \boldsymbol{\sigma})-\left(\mathbf{s}_{h}, \boldsymbol{\tau}_{h}\right)\right\|\right. \\
+\sup _{\substack{\tilde{s}_{h} \in \tilde{X}_{1, h} \\
\tilde{\mathbf{s}}_{h} \neq 0}}\left\{\frac{\left[\mathbf{F}_{1}-\mathbf{A}_{1}(\mathbf{t})-\mathbf{B}_{1}^{*}(\boldsymbol{\sigma}), \tilde{\mathbf{s}}_{h}\right]}{\left\|\tilde{\mathbf{s}}_{h}\right\|}\right\} \\
\left.+\sup _{\substack{\tilde{\boldsymbol{\tau}}_{h} \in \tilde{M}_{1, h} \\
\tilde{\boldsymbol{\tau}}_{h} \neq 0}}\left\{\frac{\left[\mathbf{G}_{1}-\mathbf{B}_{1}(\mathbf{t}), \tilde{\boldsymbol{\tau}}_{h}\right]}{\left\|\tilde{\boldsymbol{\tau}}_{h}\right\|}\right\}\right\} .
\end{aligned}
$$

On the other hand, following the usual approach from [22] and applying now the properties of the operators $\mathbf{A}$ and $\mathbf{B}$ one can prove that there exists $\bar{C}>0$, 
depending only on $\gamma,\left\|\mathbf{B}_{1}\right\|,\|\mathbf{B}\|$ and $\beta^{*}$, such that for all $h \in \mathcal{I}$ :

$$
\left\|u-u_{h}\right\| \leq \bar{C}\left\{\left\|(\mathbf{t}, \boldsymbol{\sigma})-\left(\mathbf{t}_{h}, \boldsymbol{\sigma}_{h}\right)\right\|+\inf _{v_{h} \in M_{h}}\left\|u-v_{h}\right\|\right\} .
$$

Finally, (4.11) and (4.12) yield (4.7), thus completing the proof of the theorem.

It is important to observe that if $\tilde{X}_{1, h} \subseteq \tilde{X}_{1}$, then

$$
\sup _{\substack{\tilde{s}_{h} \in \tilde{X}_{1, h} \\ \tilde{\mathbf{s}}_{h} \neq 0}}\left\{\frac{\left[\mathbf{F}_{1}-\mathbf{A}_{1}(\mathbf{t})-\mathbf{B}_{1}^{*}(\boldsymbol{\sigma}), \tilde{\mathbf{s}}_{h}\right]}{\left\|\tilde{\mathbf{s}}_{h}\right\|}\right\}=0 .
$$

Similarly, if $\tilde{M}_{1, h} \subseteq \tilde{M}_{1}$, then

$$
\sup _{\substack{\tilde{\boldsymbol{\tau}}_{h} \in \tilde{M}_{1, h} \\ \tilde{\boldsymbol{\tau}}_{h} \neq 0}}\left\{\frac{\left[\mathbf{G}_{1}-\mathbf{B}_{1}(\mathbf{t}), \tilde{\boldsymbol{\tau}}_{h}\right]}{\left\|\tilde{\boldsymbol{\tau}}_{h}\right\|}\right\}=0 .
$$

It follows that if $V_{h} \subseteq V$, then (4.7) becomes the usual Cea estimate for the Galerkin error. In other words, the second and third terms on the right hand side of (4.7) constitute the consistency error for the case in which $V_{h}$ is not a subspace of $V$.

\section{Existence, Uniqueness AND APPROXIMATION RESUlts}

5.1. The continuous problem. We now go back to our problem from Section 3. In the sequel, we show that (3.12) satisfies the hypotheses of Theorem 4.1 .

To begin with, we state the continuous inf-sup condition for $\mathbf{B}$.

Lemma 5.1. There exists $\beta>0$ such that for all $v \in M$,

$$
\sup _{\substack{(\mathbf{s}, \boldsymbol{\tau}) \in X \\(\mathbf{s}, \boldsymbol{\tau}) \neq 0}} \frac{[\mathbf{B}(\mathbf{s}, \boldsymbol{\tau}), v]}{\|(\mathbf{s}, \boldsymbol{\tau})\|_{X}} \geq \beta\|v\|_{M}
$$

Proof. We only observe that

$$
\sup _{\substack{(\mathbf{s}, \boldsymbol{\tau}) \in X \\(\mathbf{s}, \boldsymbol{\tau}) \neq 0}} \frac{[\mathbf{B}(\mathbf{s}, \boldsymbol{\tau}), v]}{\|(\mathbf{s}, \boldsymbol{\tau})\|_{X}} \geq \sup _{\substack{\boldsymbol{\tau} \in M_{1} \\ \boldsymbol{\tau} \neq 0}} \frac{-\int_{\Omega} v \operatorname{div} \boldsymbol{\tau} d x}{\|\boldsymbol{\tau}\|_{H(\operatorname{div} ; \Omega)}} .
$$

The rest of the proof is quite standard and we refer the interested reader to [22, 20] or [31].

It is important to remark that, using classical regularity results, one can show (cf. Lemma 4.4 in [31]), that there exists $\beta>0$ such that

$$
\sup _{{\boldsymbol{\tau} \in\left[H^{1}(\Omega)\right]^{2} \cap H_{0}(\mathrm{div} ; \Omega)}_{\boldsymbol{\tau} \neq 00}} \frac{-\int_{\Omega} v \operatorname{div} \boldsymbol{\tau} d x}{\|\boldsymbol{\tau}\|_{\left[H^{1}(\Omega)\right]^{2}}} \geq \beta\|v\|_{L^{2}(\Omega)} \quad \forall v \in L^{2}(\Omega) .
$$

This stronger inf-sup condition will be needed in subsection 5.2 to prove the discrete inf-sup condition for $\mathbf{B}$.

On the other hand, it is straigthforward to see that $V:=\operatorname{Ker}(\mathbf{B})=\tilde{X}_{1} \times \tilde{M}_{1}$, where

$$
\tilde{X}_{1}=X_{1} \quad \text { and } \quad \tilde{M}_{1}=\left\{\boldsymbol{\tau} \in M_{1}: \quad \operatorname{div} \boldsymbol{\tau}=0 \quad \text { in } \quad \Omega\right\} .
$$

Then, the inf-sup condition for $\mathbf{B}_{1}$ is also easily established. 
Lemma 5.2. We have

$$
\sup _{\substack{\mathbf{s} \in \tilde{X}_{1} \\ \mathbf{s} \neq 0}} \frac{\left[\mathbf{B}_{1}(\mathbf{s}), \boldsymbol{\tau}\right]}{\|\mathbf{s}\|_{X_{1}}} \geq\|\boldsymbol{\tau}\|_{H(\operatorname{div} ; \Omega)} \quad \forall \boldsymbol{\tau} \in \tilde{M}_{1} .
$$

Proof. Since $\left[\mathbf{B}_{1}(\mathbf{s}), \boldsymbol{\tau}\right]=-\int_{\Omega} \boldsymbol{\zeta} \cdot \boldsymbol{\tau} d x+\langle\lambda, \boldsymbol{\tau} \cdot \nu\rangle$ for all $\mathbf{s}:=(\boldsymbol{\zeta}, \lambda) \in \tilde{X}_{1}$, it follows that

$$
\sup _{\substack{\mathbf{s} \in \tilde{X}_{1} \\ \mathbf{s} \neq 0}} \frac{\left[\mathbf{B}_{1}(\mathbf{s}), \boldsymbol{\tau}\right]}{\|\mathbf{s}\|_{X_{1}}} \geq \sup _{\substack{\boldsymbol{\zeta}, 0) \in \tilde{X}_{1} \\ \boldsymbol{\zeta} \neq 0}} \frac{-\int_{\Omega} \boldsymbol{\zeta} \cdot \boldsymbol{\tau} d x}{\|\boldsymbol{\zeta}\|_{\left[L^{2}(\Omega)\right]^{2}}}=\|\boldsymbol{\tau}\|_{\left[L^{2}(\Omega)\right]^{2}}=\|\boldsymbol{\tau}\|_{H(\operatorname{div} ; \Omega)}
$$

for all $\boldsymbol{\tau} \in \tilde{M}_{1}$, which completes the proof.

Let us now recall that the nonlinear coefficients $a_{i}$ satisfy the assumptions (A.3) and (A.4) (cf. Section 3). Then, the following lemma establishes the strong monotonicity and Lipschitz-continuity of the nonlinear operator $\mathbf{A}_{1}: X_{1} \rightarrow X_{1}^{\prime}$.

Lemma 5.3. There exist positive constants $\alpha, \gamma$ such that

$$
\left[\mathbf{A}_{1}(\mathbf{t})-\mathbf{A}_{1}(\mathbf{s}), \mathbf{t}-\mathbf{s}\right] \geq \alpha\|\mathbf{t}-\mathbf{s}\|_{X_{1}}^{2}
$$

and

$$
\left\|\mathbf{A}_{1}(\mathbf{t})-\mathbf{A}_{1}(\mathbf{s})\right\|_{X_{1}^{\prime}} \leq \gamma\|\mathbf{t}-\mathbf{s}\|_{X_{1}}
$$

for all $\mathbf{t}, \mathbf{s} \in X_{1}$.

Proof. Let $\mathbf{t}:=(\boldsymbol{\theta}, \xi)$ and $\mathbf{s}:=(\boldsymbol{\zeta}, \lambda) \in X_{1}$. Then, we have

$$
\begin{gathered}
{\left[\mathbf{A}_{1}(\mathbf{t})-\mathbf{A}_{1}(\mathbf{s}), \mathbf{t}-\mathbf{s}\right]=\int_{\Omega_{1}}[a(\cdot, \boldsymbol{\theta})-a(\cdot, \boldsymbol{\zeta})] \cdot[\boldsymbol{\theta}-\boldsymbol{\zeta}] d x} \\
+\|\boldsymbol{\theta}-\boldsymbol{\zeta}\|_{\left[L^{2}\left(\Omega_{2}\right)\right]^{2}}^{2}+2\langle\xi-\lambda, \mathbf{W}(\xi-\lambda)\rangle,
\end{gathered}
$$

which, using the coerciveness property (2.4), yields

$$
\begin{aligned}
{\left[\mathbf{A}_{1}(\mathbf{t})-\right.} & \left.\mathbf{A}_{1}(\mathbf{s}), \mathbf{t}-\mathbf{s}\right] \geq \int_{\Omega_{1}}[a(\cdot, \boldsymbol{\theta})-a(\cdot, \boldsymbol{\zeta})] \cdot[\boldsymbol{\theta}-\boldsymbol{\zeta}] d x \\
& +\|\boldsymbol{\theta}-\boldsymbol{\zeta}\|_{\left[L^{2}\left(\Omega_{2}\right)\right]^{2}}^{2}+2 C_{0}\|\xi-\lambda\|_{H^{1 / 2}(\Gamma)}^{2} .
\end{aligned}
$$

Now, proceeding as in Section 5 of [17], we find that

$$
a_{i}(x, \boldsymbol{\theta}(x))-a_{i}(x, \boldsymbol{\zeta}(x))=\int_{0}^{1} \sum_{j=1}^{2} \frac{\partial}{\partial \hat{\theta}_{j}} a_{i}(x, \hat{\boldsymbol{\theta}}(x, t))\left[\theta_{j}(x)-\zeta_{j}(x)\right] d t,
$$

where $\hat{\boldsymbol{\theta}}(x, t):=\boldsymbol{\zeta}(x)+t[\boldsymbol{\theta}(x)-\boldsymbol{\zeta}(x)], \boldsymbol{\theta}=\left(\theta_{1}, \theta_{2}\right)$ and $\boldsymbol{\zeta}=\left(\zeta_{1}, \zeta_{2}\right)$.

Thus, applying (5.5) and (A.3), we deduce that

$$
\begin{aligned}
\int_{\Omega_{1}} & {[a(\cdot, \boldsymbol{\theta})-a(\cdot, \boldsymbol{\zeta})] \cdot[\boldsymbol{\theta}-\boldsymbol{\zeta}] d x=\sum_{i=1}^{2} \int_{\Omega_{1}}\left[a_{i}(\cdot, \boldsymbol{\theta})-a_{i}(\cdot, \boldsymbol{\zeta})\right]\left[\theta_{i}-\zeta_{i}\right] d x } \\
& =\sum_{i=1}^{2} \int_{\Omega_{1}} \int_{0}^{1} \sum_{j=1}^{2} \frac{\partial}{\partial \hat{\theta}_{j}} a_{i}(\cdot, \hat{\boldsymbol{\theta}}(\cdot, t))\left[\theta_{j}-\zeta_{j}\right]\left[\theta_{i}-\zeta_{i}\right] d t d x \\
& \geq C \sum_{i=1}^{2} \int_{\Omega_{1}}\left[\theta_{i}-\zeta_{i}\right]^{2} d x=C\|\boldsymbol{\theta}-\boldsymbol{\zeta}\|_{\left[L^{2}\left(\Omega_{1}\right)\right]^{2}}^{2}
\end{aligned}
$$


Therefore, replacing (5.6) back into (5.4) we obtain (5.2).

On the other hand, the proof of (5.3), which proceeds similarly to Section 6 of [17, again uses the relation (5.5) and applies now the assumption (A.4) and the continuity property of the boundary integral operator W. Hence, we omit further details.

Corollary 5.4. Let $V_{1}:=\left\{\mathbf{s} \in \tilde{X}_{1}:\left[\mathbf{B}_{1}(\mathbf{s}), \boldsymbol{\tau}\right]=0 \forall \boldsymbol{\tau} \in \tilde{M}_{1}\right\}$ and let $\Pi_{1}: X_{1}^{\prime} \rightarrow$ $V_{1}^{\prime}$ be the canonical imbedding. Then, for any $\tilde{\mathbf{t}} \in X_{1}$, the nonlinear operator $\Pi_{1} \mathbf{A}_{1}(\cdot+\tilde{\mathbf{t}}): V_{1} \rightarrow V_{1}^{\prime}$ is strongly monotone.

Proof. It follows straigthforwardly from the previous lemma and the fact that $V_{1} \subseteq$ $\tilde{X}_{1}=X_{1}$. Indeed, given $\tilde{\mathbf{t}} \in X_{1}, \mathbf{t}, \mathbf{s} \in V_{1}$, we have

$$
\begin{gathered}
{\left[\Pi_{1} \mathbf{A}_{1}(\mathbf{t}+\tilde{\mathbf{t}})-\Pi_{1} \mathbf{A}_{1}(\mathbf{s}+\tilde{\mathbf{t}}), \mathbf{t}-\mathbf{s}\right]} \\
=\left[\mathbf{A}_{1}(\mathbf{t}+\tilde{\mathbf{t}})-\mathbf{A}_{1}(\mathbf{s}+\tilde{\mathbf{t}}),(\mathbf{t}+\tilde{\mathbf{t}})-(\mathbf{s}+\tilde{\mathbf{t}})\right] \geq \alpha\|\mathbf{t}-\mathbf{s}\|_{X_{1}}^{2},
\end{gathered}
$$

which ends the proof.

We are now in position to provide our main result concerning the solvability of the continuous problem (3.12).

Theorem 5.5. There exists a unique $((\mathbf{t}, \boldsymbol{\sigma}), u) \in X \times M$ solution of the dual-dual mixed formulation (3.12).

Proof. By virtue of the previous results of this section, the proof follows from a direct application of the abstract Theorem 4.1

5.2. A discrete Galerkin scheme. Now, we introduce specific finite element subspaces, define the associated Galerkin scheme, and prove that the hypotheses of both Theorem 4.2 and Theorem 4.3 are satisfied.

First, given $N \in \mathbf{N}$, we let $0=t_{0}<t_{1}<\cdots<t_{N}=2 \pi$ be a uniform partition of $[0,2 \pi]$ with $t_{j+1}-t_{j}=\tilde{h}=\frac{2 \pi}{N}$ for $j \in\{0,1, \ldots, N-1\}$. Also, let $\mathbf{z}:[0,2 \pi] \rightarrow \Gamma$ be the usual parametrization of the circle $\Gamma$ given by $\mathbf{z}(t):=r(\cos (t), \sin (t))^{T}$ for all $t \in[0,2 \pi]$. We denote by $\Omega_{\tilde{h}}$ the annular domain bounded by $\Gamma_{0}$ and the polygonal line $\Gamma_{\tilde{h}}$ whose vertices are $\left\{\mathbf{z}\left(t_{1}\right), \mathbf{z}\left(t_{2}\right), \ldots, \mathbf{z}\left(t_{N}\right)\right\}$.

Let $\mathcal{T}_{\tilde{h}}$ be a regular triangulation of $\Omega_{\tilde{h}}$ by triangles $T$ of diameter $h_{T}$ such that $h:=\sup _{T \in \mathcal{T}_{\tilde{h}}} h_{T}$. For simplicity, we assume that for each $T \in \mathcal{T}_{\tilde{h}}$, either $T \subseteq \bar{\Omega}_{1}$ or $T \subseteq \bar{\Omega}_{2}$. Then, we replace each triangle $T \in \mathcal{T}_{\tilde{h}}$ with one side along $\Gamma_{\tilde{h}}$, by the corresponding curved triangle with one side along $\Gamma$. In this way, we obtain from $\mathcal{T}_{\tilde{h}}$ a triangulation $\mathcal{T}_{h}$ of $\bar{\Omega}$ made up of straight and curved triangles.

Next, we consider the canonical triangle with vertices $\hat{P}_{1}=(0,0)^{T}, \hat{P}_{2}=(1,0)^{T}$ and $\hat{P}_{3}=(0,1)^{T}$ as a reference triangle $\hat{T}$, and introduce a family of bijective mappings $\left\{F_{T}\right\}_{T \in \mathcal{T}_{h}}$, such that $F_{T}(\hat{T})=T$. In particular, if $T$ is a straight triangle of $\mathcal{T}_{h}$, then $F_{T}$ is the well known invertible affine mapping defined by $F_{T}(\hat{x})=$ $B_{T} \hat{x}+b_{T}$, where $B_{T}$, a square matrix of order 2 , and $b_{T} \in \mathbf{R}^{2}$ depend on the vertices of $T$.

Now, if $T$ is a curved triangle with vertices $P_{1}, P_{2}$ and $P_{3}$, such that $P_{2}=$ $\mathbf{z}\left(t_{j-1}\right) \in \Gamma$ and $P_{3}=\mathbf{z}\left(t_{j}\right) \in \Gamma$, then $F_{T}(\hat{x})=B_{T} \hat{x}+b_{T}+G_{T}(\hat{x})$ for all $\hat{x}:=$ $\left(\hat{x}_{1}, \hat{x}_{2}\right) \in \hat{T}$, where

$$
G_{T}(\hat{x})=\frac{\hat{x}_{1}}{1-\hat{x}_{2}}\left\{\mathbf{z}\left(t_{j-1}+\hat{x}_{2}\left(t_{j}-t_{j-1}\right)\right)-\left[\mathbf{z}\left(t_{j-1}\right)+\hat{x}_{2}\left(\mathbf{z}\left(t_{j}\right)-\mathbf{z}\left(t_{j-1}\right)\right)\right]\right\} .
$$


It can be proved (see, e.g., Theorem 22.4 in [35]) that $F_{T}$ is a diffeomorphism of class $C^{\infty}$ that maps one-to-one $\hat{T}$ onto the curved triangle $T$ in such a way that $F_{T}\left(\hat{P}_{i}\right)=P_{i}$ for $i \in\{1,2,3\}$. Also, the image of edge $\hat{P}_{2} \hat{P}_{3}$ is the curved side of $T$ and, since $G_{T}(\hat{x})=(0,0)^{T}$ for $\hat{x}_{1}=0$ and for $\hat{x}_{2}=0$, the two other edges of $\hat{T}$ are transformed linearly under $F_{T}$ to the straight sides of $T$.

We now consider the lowest order Raviart-Thomas spaces. For this purpose, we first let

$$
\mathcal{R} T_{0}(\hat{T}):=\operatorname{span}\left\{\left(\begin{array}{l}
1 \\
0
\end{array}\right),\left(\begin{array}{l}
0 \\
1
\end{array}\right),\left(\begin{array}{l}
\hat{x}_{1} \\
\hat{x}_{2}
\end{array}\right)\right\},
$$

and for each triangle $T \in \mathcal{T}_{h}$, we put

$$
\mathcal{R} T_{0}(T):=\left\{\boldsymbol{\tau}: \quad \boldsymbol{\tau}=J\left(F_{T}\right)^{-1}\left(D F_{T}\right) \hat{\boldsymbol{\tau}} \circ F_{T}^{-1}, \hat{\boldsymbol{\tau}} \in \mathcal{R} T_{0}(\hat{T})\right\},
$$

where $J\left(F_{T}\right)$ and $D\left(F_{T}\right)$ denote, respectively, the jacobian and the Frêchet differential of the mapping $F_{T}$.

Then, we define the finite element subspaces for the unknowns $\boldsymbol{\theta}$ and $\boldsymbol{\sigma}$, as follows:

$$
X_{1, h}^{\boldsymbol{\theta}}:=\left\{\boldsymbol{\tau}_{h} \in\left[L^{2}(\Omega)\right]^{2}:\left.\quad \boldsymbol{\tau}_{h}\right|_{T} \in \mathcal{R} T_{0}(T) \quad \forall T \in \mathcal{T}_{h}\right\}
$$

and

$$
M_{1, h}:=X_{1, h}^{\boldsymbol{\theta}} \cap H_{0}(\operatorname{div} ; \Omega) .
$$

Note that $X_{1, h}^{\boldsymbol{\theta}}$ does not require continuity of the normal components through the sides of each triangle $T$, while $M_{1, h}$ certainly does.

Next, we set

$$
\begin{aligned}
H_{h}(0,2 \pi):= & \left\{\tilde{\lambda}_{h}:[0,2 \pi] \rightarrow \mathbf{R}, \quad \tilde{\lambda}_{h} \text { is continuous and periodic of period } 2 \pi\right. \\
& \left.\left.\tilde{\lambda}_{h}\right|_{\left[t_{j-1}, t_{j}\right]} \in \mathbf{P}_{1}\left(t_{j-1}, t_{j}\right) \quad \forall j \in\{1, \ldots, N\}, \quad \int_{0}^{2 \pi} \tilde{\lambda}_{h}(t) d t=0\right\}
\end{aligned}
$$

and define the finite element subspace for the unknown $\xi$ :

$$
X_{1, h}^{\xi}:=\left\{\lambda_{h}: \Gamma \rightarrow \mathbf{R}, \quad \lambda_{h}=\tilde{\lambda}_{h} \circ \mathbf{z}^{-1}, \tilde{\lambda}_{h} \in H_{h}(0,2 \pi)\right\} .
$$

Hereafter, given a non-negative integer $k$ and a subset $S$ of $\mathbf{R}$ or $\mathbf{R}^{2}, \mathbf{P}_{k}(S)$ denotes the space of polynomials defined on $S$ of degree $\leq k$. At this point we remark that the simplicity of the definition of $X_{1, h}^{\xi}$ is due to the fact that $\Gamma$ is a circle, which yields a constant jacobian of the transformation $\mathbf{z}$. If this were not the case, then one should proceed differently (see, e.g., 30]).

Note that $H_{h}(0,2 \pi) \subseteq H^{1 / 2}[0,2 \pi]$, where for each $p>0, H^{p}[0,2 \pi]$ denotes the usual Sobolev space of $2 \pi$-periodic functions (see, e.g., Section 8.2 of [29]). Hence, according to the regularity of $\mathbf{z}$ and the definition of the Sobolev spaces on the boundary $\Gamma$ (see, e.g., Section 8.3 in [29]), we deduce that $X_{1, h}^{\xi} \subseteq H_{0}^{1 / 2}(\Gamma)$. Finally, we put

$$
\begin{aligned}
X_{1, h} & :=X_{1, h}^{\boldsymbol{\theta}} \times X_{1, h}^{\xi}, \\
X_{h} & :=X_{1, h} \times M_{1, h},
\end{aligned}
$$


and consider the piecewise constant functions as the finite element subspace for the unknown $u$, that is

$$
M_{h}:=\left\{v_{h} \in L^{2}(\Omega):\left.\quad v_{h}\right|_{T} \in \mathbf{P}_{0}(T) \quad \forall T \in \mathcal{T}_{h}\right\} .
$$

In this way, the Galerkin scheme associated with the continuous problem (3.12) reads as follows: Find $\left(\left(\mathbf{t}_{h}, \boldsymbol{\sigma}_{h}\right), u_{h}\right) \in X_{h} \times M_{h}$ such that

$$
\begin{aligned}
{\left[\mathbf{A}\left(\mathbf{t}_{h}, \boldsymbol{\sigma}_{h}\right),\left(\mathbf{s}_{h}, \boldsymbol{\tau}_{h}\right)\right]+\left[\mathbf{B}^{*}\left(u_{h}\right),\left(\mathbf{s}_{h}, \boldsymbol{\tau}_{h}\right)\right] } & =0, \\
{\left[\mathbf{B}\left(\mathbf{t}_{h}, \boldsymbol{\sigma}_{h}\right), v_{h}\right] } & =\left[\mathbf{G}, v_{h}\right],
\end{aligned}
$$

for all $\left(\left(\mathbf{s}_{h}, \boldsymbol{\tau}_{h}\right), v_{h}\right) \in X_{h} \times M_{h}$.

In what follows, we verify that the introduced finite element subspaces above satisfy the corresponding discrete inf-sup conditions.

Lemma 5.6. There exists $\beta^{*}>0$, independent of the subspaces involved, such that for all $v_{h} \in M_{h}$

$$
\sup _{\substack{\left(\mathbf{s}_{h}, \boldsymbol{\tau}_{h}\right) \in X_{h} \\\left(\mathbf{s}_{h}, \boldsymbol{\tau}_{h}\right) \neq 0}} \frac{\left[\mathbf{B}\left(\mathbf{s}_{h}, \boldsymbol{\tau}_{h}\right), v_{h}\right]}{\left\|\left(\mathbf{s}_{h}, \boldsymbol{\tau}_{h}\right)\right\|_{X}} \geq \beta^{*}\left\|v_{h}\right\|_{M} .
$$

Proof. We proceed similarly as in Lemma 4.3 of [31]. First, we observe that

$$
\sup _{\substack{\left(\mathbf{s}_{h}, \boldsymbol{\tau}_{h}\right) \in X_{h} \\\left(\mathbf{s}_{h}, \boldsymbol{\tau}_{h}\right) \neq 0}} \frac{\left[\mathbf{B}\left(\mathbf{s}_{h}, \boldsymbol{\tau}_{h}\right), v_{h}\right]}{\left\|\left(\mathbf{s}_{h}, \boldsymbol{\tau}_{h}\right)\right\|_{X}} \geq \sup _{\substack{\boldsymbol{\tau}_{h} \in M_{1, h} \\ \boldsymbol{\tau}_{h} \neq 0}} \frac{-\int_{\Omega} v_{h} \operatorname{div} \boldsymbol{\tau}_{h} d x}{\left\|\boldsymbol{\tau}_{h}\right\|_{H(\operatorname{div} ; \Omega)}} .
$$

Then, we introduce the equilibrium interpolation operator (cf. [2], [33]) $E_{h}$ : $\left[H^{1}(\Omega)\right]^{2} \rightarrow X_{1, h}^{\boldsymbol{\theta}} \cap H(\operatorname{div} ; \Omega)$, which is characterized on each $T \in \mathcal{T}_{h}$ by

$$
\int_{e}\left(E_{h} \boldsymbol{\tau}\right) \cdot \nu d s=\int_{e} \boldsymbol{\tau} \cdot \nu d s \text { for all edges } e \text { of } T .
$$

It is well known that $E_{h}$ satisfies the commuting diagram property

$$
\operatorname{div}\left(E_{h} \boldsymbol{\tau}\right)=\mathcal{P}_{h}(\operatorname{div} \boldsymbol{\tau}) \quad \forall \boldsymbol{\tau} \in\left[H^{1}(\Omega)\right]^{2},
$$

where $\mathcal{P}_{h}$ is the orthogonal projection from $L^{2}(\Omega)$ onto the subspace $M_{h}$. In other words,

$$
\int_{\Omega} \operatorname{div}\left(E_{h} \boldsymbol{\tau}\right) v_{h} d x=\int_{\Omega} v_{h} \operatorname{div} \boldsymbol{\tau} d x \quad \forall v_{h} \in M_{h},
$$

and also, from (5.15) we get

$$
\int_{\Gamma}\left(E_{h} \boldsymbol{\tau}\right) \cdot \nu d s=\int_{\Gamma} \boldsymbol{\tau} \cdot \nu d s \quad \forall \boldsymbol{\tau} \in\left[H^{1}(\Omega)\right]^{2} .
$$

It follows that $\left(E_{h} \boldsymbol{\tau}\right) \in X_{1, h}^{\boldsymbol{\theta}} \cap H_{0}(\operatorname{div} ; \Omega)$ for all $\boldsymbol{\tau} \in\left[H^{1}(\Omega)\right]^{2} \cap H_{0}(\operatorname{div} ; \Omega)$. Moreover, using (5.16) and the approximation properties of $E_{h}$ (cf. [2] or [33]), we conclude that the family of operators $E_{h}:\left[H^{1}(\Omega)\right]^{2} \cap H_{0}(\operatorname{div} ; \Omega) \rightarrow M_{1, h}$ is uniformly bounded. This means that there exists $\tilde{C}>0$, independent of $h \in \mathcal{I}$, such that

$$
\left\|E_{h} \boldsymbol{\tau}\right\|_{H(\operatorname{div} ; \Omega)} \leq \tilde{C}\|\boldsymbol{\tau}\|_{\left[H^{1}(\Omega)\right]^{2}} \quad \forall \boldsymbol{\tau} \in\left[H^{1}(\Omega)\right]^{2} \cap H_{0}(\operatorname{div} ; \Omega) .
$$


Now, going back to (5.14), using (5.17), (5.18) and the stronger continuous infsup condition (5.1), we can write

$$
\begin{aligned}
& \sup _{\substack{\boldsymbol{\tau}_{h} \in M_{1, h} \\
\boldsymbol{\tau}_{h} \neq 0}} \frac{-\int_{\Omega} v_{h} \operatorname{div} \boldsymbol{\tau}_{h} d x}{\left\|\boldsymbol{\tau}_{h}\right\|_{H(\operatorname{div} ; \Omega)}} \geq \sup _{\substack{\boldsymbol{\tau} \in\left[H^{1}(\Omega)\right]^{2} \cap H_{0}(\operatorname{div} ; \Omega) \\
\boldsymbol{\tau} \neq 0}} \frac{\int_{\Omega} v_{h} \operatorname{div}\left(E_{h} \boldsymbol{\tau}\right) d x}{\left\|E_{h} \boldsymbol{\tau}\right\|_{H(\operatorname{div} ; \Omega)}} \\
& \quad \geq \frac{1}{\tilde{C}} \sup _{\substack{\boldsymbol{\tau} \in\left[H^{1}(\Omega)\right]^{2} \cap H_{0}(\mathrm{div} ; \Omega) \\
\boldsymbol{\tau} \neq 0}} \frac{\int_{\Omega} v_{h} \operatorname{div} \boldsymbol{\tau} d x}{\|\boldsymbol{\tau}\|_{\left[H^{1}(\Omega)\right]^{2}}} \geq \beta^{*}\left\|v_{h}\right\|_{M},
\end{aligned}
$$

which finishes the proof.

In order to continue our analysis, we need to characterize the discrete kernel $V_{h}$.

Lemma 5.7. We have $V_{h}=\tilde{X}_{1, h} \times \tilde{M}_{1, h}$, where $\tilde{X}_{1, h}=X_{1, h}$ and

$$
\tilde{M}_{1, h}:=\left\{\boldsymbol{\tau}_{h} \in M_{1, h}: \quad \operatorname{div} \boldsymbol{\tau}_{h}=0 \quad \text { in } \quad \Omega\right\} .
$$

Proof. According to the definition of $\mathbf{B}$, we have

$$
V_{h}=\left\{\left(\mathbf{s}_{h}, \boldsymbol{\tau}_{h}\right) \in X_{h}: \quad \int_{\Omega} v_{h} \operatorname{div} \boldsymbol{\tau}_{h} d x=0 \forall v_{h} \in M_{h}\right\},
$$

and then $V_{h}=\tilde{X}_{1, h} \times \tilde{M}_{1, h}$, with $\tilde{X}_{1, h}=X_{1, h}$ and

$$
\tilde{M}_{1, h}=\left\{\boldsymbol{\tau}_{h} \in M_{1, h}: \quad \int_{\Omega} v_{h} \operatorname{div} \boldsymbol{\tau}_{h} d x=0 \forall v_{h} \in M_{h}\right\} .
$$

Now, given $\boldsymbol{\tau}_{h} \in \tilde{M}_{1, h}$ and $T \in \mathcal{T}_{h}$, we may choose $v_{h} \in M_{h}$ such that $\left.v_{h}\right|_{T} \equiv 1$ and $v_{h} \equiv 0$ in $\Omega-T$. It follows that $\int_{T} \operatorname{div} \boldsymbol{\tau}_{h} d x=0$ for all $T \in \mathcal{T}_{h}$. But, using Lemma 1.5 (identity (1.49)) in Chapter III of [2, we can write

$$
0=\int_{T} \operatorname{div} \boldsymbol{\tau}_{h} d x=\int_{\hat{T}} \operatorname{div} \hat{\boldsymbol{\tau}}_{h, T} d \hat{x},
$$

where $\hat{\boldsymbol{\tau}}_{h, T} \in \mathcal{R} T_{0}(\hat{T})$ is such that $\left.\boldsymbol{\tau}_{h}\right|_{T}=J\left(F_{T}\right)^{-1}\left(D F_{T}\right) \hat{\boldsymbol{\tau}}_{h, T} \circ F_{T}^{-1}$.

Since $\operatorname{div} \hat{\boldsymbol{\tau}}_{h, T}$ is constant in $\hat{T}$, we deduce from (5.19) that $\operatorname{div} \hat{\boldsymbol{\tau}}_{h, T}=0$ for all $T \in \mathcal{T}_{h}$, and therefore, applying the identity (1.47) in Chapter III of [2], we conclude that

$$
\operatorname{div}\left(\left.\boldsymbol{\tau}_{h}\right|_{T}\right)=J\left(F_{T}\right)^{-1} \operatorname{div} \hat{\boldsymbol{\tau}}_{h, T}=0 \quad \forall T \in \mathcal{T}_{h} .
$$

This completes the proof.

We now prove the discrete inf-sup condition for $\mathbf{B}_{1}$.

Lemma 5.8. There exists $\beta_{1}^{*}>0$, independent of the subspaces involved, such that for all $\boldsymbol{\tau}_{h} \in \tilde{M}_{1, h}$

$$
\sup _{\substack{\mathbf{s}_{h} \in \tilde{X}_{1, h} \\ \mathbf{s}_{h} \neq 0}} \frac{\left[\mathbf{B}_{1}\left(\mathbf{s}_{h}\right), \boldsymbol{\tau}_{h}\right]}{\left\|\mathbf{s}_{h}\right\|_{X_{1}}} \geq \beta_{1}^{*}\left\|\boldsymbol{\tau}_{h}\right\|_{M_{1}}
$$


Proof. Since $\tilde{X}_{1, h}=X_{1, h}=X_{1, h}^{\boldsymbol{\theta}} \times X_{1, h}^{\xi}$, we have for all $\boldsymbol{\tau}_{h} \in \tilde{M}_{1, h}$

$$
\sup _{\substack{\mathbf{s}_{h} \in \tilde{X}_{1, h} \\ \mathbf{s}_{h} \neq 0}} \frac{\left[\mathbf{B}_{1}\left(\mathbf{s}_{h}\right), \boldsymbol{\tau}_{h}\right]}{\left\|\mathbf{s}_{h}\right\|_{X_{1}}} \geq \sup _{\substack{\boldsymbol{\zeta}_{h} \in X_{1, h} \\ \boldsymbol{\zeta}_{h} \neq 0}} \frac{-\int_{\Omega} \boldsymbol{\tau}_{h} \cdot \boldsymbol{\zeta}_{h} d x}{\left\|\boldsymbol{\zeta}_{h}\right\|_{\left[L^{2}(\Omega)\right]^{2}}} .
$$

Then, using that $\tilde{M}_{1, h} \subseteq M_{1, h} \subseteq X_{1, h}^{\boldsymbol{\theta}}$, we deduce that

$$
\sup _{\substack{\boldsymbol{\zeta}_{h} \in X_{1, h}^{\boldsymbol{\theta}} \\ \boldsymbol{\zeta}_{h} \neq 0}} \frac{-\int_{\Omega} \boldsymbol{\tau}_{h} \cdot \boldsymbol{\zeta}_{h} d x}{\left\|\boldsymbol{\zeta}_{h}\right\|_{\left[L^{2}(\Omega)\right]^{2}}}=\left\|\boldsymbol{\tau}_{h}\right\|_{\left[L^{2}(\Omega)\right]^{2}}=\left\|\boldsymbol{\tau}_{h}\right\|_{H(\mathrm{div} ; \Omega)},
$$

where the last equality follows from the characterization of $\tilde{M}_{1, h}$ given in Lemma 5.7. This ends the proof.

The unique solvability of the Galerkin scheme (15.13) and the corresponding error estimate can be established now.

Theorem 5.9. There exists a unique $\left(\left(\mathbf{t}_{h}, \boldsymbol{\sigma}_{h}\right), u_{h}\right) \in X_{h} \times M_{h}$ solution of the Galerkin scheme (5.13). In addition, there exists $C>0$, independent of $h$, such that the following Cea estimate holds

$$
\begin{gathered}
\left\|((\mathbf{t}, \boldsymbol{\sigma}), u)-\left(\left(\mathbf{t}_{h}, \boldsymbol{\sigma}_{h}\right), u_{h}\right)\right\| \\
\leq C \inf _{\left(\left(\mathbf{s}_{h}, \boldsymbol{\tau}_{h}\right), v_{h}\right) \in X_{h} \times M_{h}}\left\|((\mathbf{t}, \boldsymbol{\sigma}), u)-\left(\left(\mathbf{s}_{h}, \boldsymbol{\tau}_{h}\right), v_{h}\right)\right\| .
\end{gathered}
$$

Proof. We first observe that Lemma 5.3 and Corollary 5.4 guarantee that the discrete operator $\mathbf{A}_{1, h}: X_{1} \rightarrow X_{1, h}^{\prime}$ is also Lipschitz-continuous, and that the family of operators $\left\{\Pi_{1, h} \mathbf{A}_{1, h}(\cdot+\tilde{\mathbf{t}}): \quad \tilde{\mathbf{t}} \in X_{1, h}, h \in \mathcal{I}\right\}$ is uniformly strongly monotone. Here, $\mathbf{A}_{1, h}$ and $\Pi_{1, h}$ are defined as in Theorem 4.2, In addition, from Lemma 5.7 and the definition of $V$ given in subsection 5.1, we deduce that $V_{h} \subseteq V$.

Therefore, by virtue of Lemmas 5.6 and 5.8 , a direct application of the abstract Theorems 4.2 and 4.3 finishes the proof.

As a consequence of the Cea estimate given by the previous theorem, we deduce the following error bound.

Theorem 5.10. Let $((\mathbf{t}, \boldsymbol{\sigma}), u)$ and $\left(\left(\mathbf{t}_{h}, \boldsymbol{\sigma}_{h}\right), u_{h}\right)$ be the unique solutions of (3.12) and (5.13), respectively, with $\mathbf{t}:=(\boldsymbol{\theta}, \xi)$ and $\mathbf{t}_{h}:=\left(\boldsymbol{\theta}_{h}, \xi_{h}\right)$. In addition, assume that $\left.\boldsymbol{\theta}\right|_{T} \in\left[H^{1}(T)\right]^{2} \forall T \in \mathcal{T}_{h}, \xi \in H^{3 / 2}(\Gamma), \boldsymbol{\sigma} \in\left[H^{1}(\Omega)\right]^{2}$, div $\boldsymbol{\sigma} \in H^{1}(\Omega)$ and $u \in H^{1}(\Omega)$. Then, there exists $\tilde{C}>0$, independent of $h$, such that the following estimate holds

$$
\begin{aligned}
& \left\|(\boldsymbol{\theta}, \xi, \boldsymbol{\sigma}, u)-\left(\boldsymbol{\theta}_{h}, \xi_{h}, \boldsymbol{\sigma}_{h}, u_{h}\right)\right\| \\
& \leq \tilde{C} h\left\{\sum_{T \in \mathcal{T}_{h}}\|\boldsymbol{\theta}\|_{\left[H^{1}(T)\right]^{2}}^{2}+\|\xi\|_{H^{3 / 2}(\Gamma)}^{2}\right. \\
& \left.\quad+\|\boldsymbol{\sigma}\|_{\left[H^{1}(\Omega)\right]^{2}}^{2}+\|\operatorname{div} \boldsymbol{\sigma}\|_{H^{1}(\Omega)}^{2}+\|u\|_{H^{1}(\Omega)}^{2}\right\}^{1 / 2}
\end{aligned}
$$


Proof. Let us denote by $E_{h, T}$ the restriction of the equilibrium operator $E_{h}$ to a given triangle $T$ of $\mathcal{T}_{h}$. We also introduce the Lagrange interpolation operator $\mathcal{L}_{h}$ from $H^{3 / 2}[0,2 \pi]$ onto $H_{h}(0,2 \pi)$. Further, as before, let $\mathcal{P}_{h}$ be the orthogonal projection from $L^{2}(\Omega)$ onto $M_{h}$.

Then from Theorem 5.9 and using again the commuting diagram property (5.16), we deduce that

$$
\begin{aligned}
\left\|(\boldsymbol{\theta}, \xi, \boldsymbol{\sigma}, u)-\left(\boldsymbol{\theta}_{h}, \xi_{h}, \boldsymbol{\sigma}_{h}, u_{h}\right)\right\|^{2} \\
\leq C\left\{\sum_{T \in \mathcal{T}_{h}}\left\|\boldsymbol{\theta}-E_{h, T} \boldsymbol{\theta}\right\|_{\left[L^{2}(T)\right]^{2}}^{2}+\left\|\left[(\xi \circ \mathbf{z})-\mathcal{L}_{h}(\xi \circ \mathbf{z})\right] \circ \mathbf{z}^{-1}\right\|_{H^{1 / 2}(\Gamma)}^{2}\right. \\
\left.\quad+\left\|\boldsymbol{\sigma}-E_{h} \boldsymbol{\sigma}\right\|_{\left[L^{2}(\Omega)\right]^{2}}^{2}+\left\|\operatorname{div} \boldsymbol{\sigma}-\mathcal{P}_{h}(\operatorname{div} \boldsymbol{\sigma})\right\|_{L^{2}(\Omega)}^{2}+\left\|u-\mathcal{P}_{h} u\right\|_{L^{2}(\Omega)}^{2}\right\} .
\end{aligned}
$$

Thus, the result follows from classical error estimates for interpolation and projection operators in the corresponding Sobolev spaces. In particular, for the second term, and using again the definition of the Sobolev spaces on $\Gamma$ through the parametrization $\mathbf{z}$ (see Section 8.3 in [29]), we obtain

$$
\begin{aligned}
\left\|\left[(\xi \circ \mathbf{z})-\mathcal{L}_{h}(\xi \circ \mathbf{z})\right] \circ \mathbf{z}^{-1}\right\|_{H^{1 / 2}(\Gamma)} & \leq C\left\|(\xi \circ \mathbf{z})-\mathcal{L}_{h}(\xi \circ \mathbf{z})\right\|_{H^{1 / 2}[0,2 \pi]} \\
& \leq C h\|\xi \circ \mathbf{z}\|_{H^{3 / 2}[0,2 \pi]} \leq C h\|\xi\|_{H^{3 / 2}(\Gamma)} .
\end{aligned}
$$

Since the other estimates are straightforward, we omit further details.

We end this paper by remarking that efficient numerical algorithms for solving discrete schemes of dual-dual structure, which are based on minimum residual and conjugate gradient methods, are provided in [13, 14] and [15.

\section{ACKNOWLEDGMENTS}

This research was completed while G. N. Gatica was visiting the Departamento de Matemáticas of the Universidad de Oviedo, España, between February 15 and March 17, 1999. This author expresses his gratitude to the colleagues of that department for their great hospitality.

\section{REFERENCES}

[1] G.R. Barrenechea, G.N. Gatica And J.-M. Thomas, Primal mixed formulations for the coupling of FEM and BEM. Part I: Linear problems, Numerical Functional Analysis and Optimization, 19 (1998), pp. 7-32. MR 99d:65310

[2] F. Brezzi And M. Fortin, Mixed and Hybrid Finite Element Methods, Springer-Verlag, 1991. MR 92d:65187

[3] U. Brink, C. Carstensen And E. Stein, Symmetric coupling of boundary elements and Raviart-Thomas-type mixed finite elements in elastostatics, Numerische Mathematik, 75 (1996), pp. 153-174. MR 98g:65106

[4] C. Carstensen and E.P. Stephan, Adaptive coupling of boundary elements and finite elements, Mathematical Modelling and Numerical Analysis, 29 (1995), pp. 779-817. MR 97e:65134

[5] M. Costabel, Boundary integral operators on Lipschitz domains: Elementary results, SIAM Journal on Mathematical Analysis, 19 (1988), pp. 613-621. MR 89h:35090

[6] M. Costabel And E.P. Stephan, Coupling of finite and boundary element methods for an elasto-plastic interface problem, SIAM Journal on Numerical Analysis, 27(5) (1990), pp. 1212-1226. MR 92c:65125 
[7] M. Feistauer, Mathematical and numerical study of nonlinear problems in fluid mechanics. In Proc. Conf. Equadiff 6, edited by J. Vosmansky and M. Zlámal, Brno 1985, Springer, Berlin, pp. 3-16. MR 88f:76002

[8] M. Feistauer, On the finite element approximation of a cascade flow problem, Numerische Mathematik, 50 (1987), pp. 655-684. MR 88h:65205

[9] K. Feng, Finite element method and natural boundary reduction. In Proc. Inter. Congress of Mathematics, Polish Academy of Sciences, Warsaw 1983, pp. 1439-1453. MR 87i:65181

[10] G.N. Gatica, On the Coupling of Boundary Integral and Finite Element Methods for Nonlinear Boundary Value Problems, Ph.D. Dissertation, University of Delaware, (1989).

[11] G.N. Gatica, Combination of mixed finite element and Dirichlet-to-Neumann methods in nonlinear plane elasticity, Applied Mathematics Letters, 10 (1997), pp. 29-35. MR 98g:73039

[12] G.N. Gatica, Solvability and Galerkin approximations of a class of nonlinear operator equations, Technical Report 99-03, Departamento de Ingeniería Matemática, Universidad de Concepción, (1999). http://www.ing-mat.udec.cl/inf-loc-dim.html

[13] G.N. Gatica AND N. Heuer, Minimum residual iteration for a dual-dual mixed formulation of exterior transmission problems, Technical Report 99-07, Departamento de Ingeniería Matemática, Universidad de Concepción, (1999). http://www.ing-mat.udec.cl/inf-loc-dim.html

[14] G.N. Gatica AND N. Heuer, An expanded mixed finite element approach via a dual-dual formulation and the minimum residual method, Technical Report 9908, Departamento de Ingeniería Matemática, Universidad de Concepción, (1999). http://www.ing-mat.udec.cl/inf-loc-dim.html. To appear in Journal of Computational and Applied Mathematics.

[15] G.N. Gatica AND N. Heuer, Conjugate gradient method for dual-dual mixed formulations, Technical Report 99-16, Departamento de Ingeniería Matemática, Universidad de Concepción, (1999). http://www.ing-mat.udec.cl/inf-loc-dim.html

[16] G.N. Gatica And G.C. HsiaO, On a class of variational formulations for some nonlinear interface problems, Rendiconti di Matematica e delle sue Applicazione, 10(4) (1990), pp. 681-715. MR 92m:35089

[17] G.N. Gatica And G.C. Hsiao, On the coupled BEM and FEM for a nonlinear exterior Dirichlet problem in $\mathbf{R}^{2}$, Numerische Mathematik, 61 (1992), pp. 171-214. MR 92j:65179

[18] G.N. Gatica and G.C. Hsiao, Boundary-Field Equation Methods for a Class of Nonlinear Problems, Pitman Research Notes in Mathematics Series, vol. 331, Longman, 1995. MR 97k:65269

[19] G.N. Gatica And G.C. Hsiao, The uncoupling of boundary integral and finite element methods for nonlinear boundary value problems, Journal of Mathematical Analysis and Applications, 189 (1995), pp. 442-461. MR 96b:65110

[20] G.N. Gatica and W.L. Wendland, Coupling of mixed finite elements and boundary elements for linear and nonlinear elliptic problems, Applicable Analysis, 63 (1996), pp. 39-75. MR 99a:65167

[21] G.N. Gatica And W.L. Wendland, Coupling of mixed finite elements and boundary elements for a hyperelastic interface problem, SIAM Journal on Numerical Analysis, 34 (1997), pp. 2335-2356. MR 98i:65098

[22] V. Girault and P.A. Raviart, Finite Element Approximation of the Navier-Stokes Equations: Theory and Algorithms, Springer, Berlin Heidelberg New York, 1986.

[23] D. Givoli, Numerical Methods for Problems in Infinite Domains. Elsevier Science Publishers B.V. (1992), Studies in Applied Mechanics 33. MR 94j:65003

[24] H. HAN AND X. WU, The approximation of the exact boundary conditions at an artificial boundary for linear elastic equations and its application, Mathematics of Computation, 59 (1992), pp. 21-37. MR 92k:35076

[25] H. HAN AND W. BAO, The artificial boundary conditions for incompressible materials on an unbounded domain, Numerische Mathematik, 77 (1997), pp. 347-363. MR 98f:73037

[26] B. HeISE, Nonlinear field calculations with multigrid Newton methods, Impact of Computing in Science and Engineering, 5 (1993), pp. 75-110. MR 95a:78002

[27] B. HeIsE, Analysis of a fully discrete finite element method for a nonlinear magnetic field problem, SIAM Journal on Numerical Analysis, 31(3) (1994), pp. 745-759. MR 95i:65156 
[28] G.C. Hsiao And S. Zhang, Optimal order multigrid methods for solving exterior boundary value problems, SIAM Journal on Numerical Analysis, 31(3) (1994), pp. 680-694. MR 95e:65116

[29] R. KRESS, Linear Integral Equations, Springer-Verlag, 1989. MR 90j:45001

[30] S. Meddahi, An optimal iterative process for the Johnson-Nedelec method of coupling boundary and finite elements, SIAM Journal on Numerical Analysis, 35(4) (1998), pp. 1393-1415. MR 99f:65173

[31] S. Meddahi, J. Valdés, O. Menéndez, And P. PÉrez, On the coupling of boundary integral and mixed finite element methods, Journal of Computational and Applied Mathematics, 69 (1996), pp. 113-124. MR 97e:65137

[32] J. NEČAS, Introduction to the Theory of Nonlinear Elliptic Equation, John Wiley \& Sons, 1986. MR 87m:35053

[33] J.E. Roberts and J.-M. Thomas, Mixed and Hybrid Methods, in Handbook of Numerical Analysis, edited by P.G. Ciarlet and J.L. Lions, vol. II, Finite Element Methods (Part 1), 1991, North-Holland, Amsterdam. CMP 91:14

[34] E.P. Stephan, Coupling of finite elements and boundary elements for some nonlinear interface problems, Computer Methods in Applied Mechanics and Engineering, 101 (1992), pp. 61-72. MR 93j:73019

[35] A. ŽEnišek, Nonlinear Elliptic and Evolution Problems and their Finite Element Approximations, Academic Press, London, 1990. MR 92c:65003

Departamento de Ingeniería Matemática, Universidad de Concepción, Casilla 160-C, Concepción, Chile

E-mail address: ggatica@ing-mat.udec.cl

Departamento de Matemáticas, Universidad de Oviedo, Calvo Sotelo s/n, 33007 Oviedo, España

E-mail address: salim@orion.ciencias.uniovi.es 